\title{
Adverse effects of organophosphorus pesticides on the liver: a brief summary of four decades of research
}

\author{
Somayyeh Karami-Mohajeri ${ }^{1,2}$, Ahmad Ahmadipour ${ }^{2}$, Hamid-Reza Rahimi ${ }^{1,2}$, and \\ Mohammad Abdollahi ${ }^{3,4}$
}

Pharmaceutics Research Center, Institute of Neuropharmacology ${ }^{1}$, Department of Toxicology and Pharmacology, Faculty of Pharmacy², Kerman University of Medical Sciences, Kerman, Pharmaceutical Sciences Research Center ${ }^{3}$, Department of Toxicology and Pharmacology, Faculty of Pharmacy, Tehran University of Medical Sciences, Tehran, Iran

[Received in May 2017; Similarity Check in May 2017; Accepted in December 2017]

\begin{abstract}
Organophosphorus pesticides (OPs) are widely used volatile pesticides that have harmful effects on the liver in acute and chronic exposures. This review article summarises and discusses a wide collection of studies published over the last 40 years reporting on the effects of OPs on the liver, in an attempt to propose general mechanisms of OP hepatotoxicity and possible treatment. Several key biological processes have been reported as involved in OP-induced hepatotoxicity such as disturbances in the antioxidant defence system, oxidative stress, apoptosis, and mitochondrial and microsomal metabolism. Most studies show that antioxidants can attenuate oxidative stress and the consequent changes in liver function. However, few studies have examined the relationship between OP structures and the severity and mechanism of their action. We hope that future in vitro, in vivo, and clinical trials will answer the remaining questions about the mechanisms of OP hepatotoxicity and its management.
\end{abstract}

KEY WORDS: apoptosis; hepatotoxicity; mitochondrial metabolism; molecular toxicity, organophosphorus; oxidative stress

Organophosphorus pesticides (OPs) have harmful effects on human health through environmental or occupational exposure. Roughly $0.1 \%$ of the applied pesticides reach the target pests, and the rest spreads through water, soil, and food (1-3). These pesticides are readily available on the market. Suicidal poisoning with OPs is common, particularly in rural areas (4). Acute poisoning with OPs is a global threat to human health that causes more than 100,000 deaths a year $(5,6)$.

They primarily affect the nervous system of the exposed organisms by inhibiting acetylcholinesterase (AChE) and raising acetylcholine levels in the cholinergic synapse. Beside cholinergic effects, OPs induce oxidative stress (7, 8), affect metabolic pathways (9), and cause multiple organ dysfunctions such as hypoxia and inadequate tissue perfusion of the liver and heart (10). In the liver they cause ultrastructural, biochemical, metabolic, and mitochondrial damage, evidenced by changes in hepatic biomarkers such as serum aminotransferase and direct and indirect bilirubin (11-31).

Their mechanisms of action on the liver and metabolism have not yet been fully clarified, and finding an effective therapy against OPs still remains a major challenge.

Correspondence to: Mohammad Abdollahi, Department of Toxicology and Pharmacology, Faculty of Pharmacy and Pharmaceutical Sciences Research Center, Tehran University of Medical Sciences, Tehran, Iran e-mail:mohammad@tums.ac.ir
This review article summarises and discusses a wide collection of studies published over the last 40 years reporting on the effects of OPs on the liver, in an attempt to propose general mechanisms of OP hepatotoxicity and possible treatment.

\section{Literature collection}

To screen for and select relevant literature we ran the keywords "Organophosphate", "organophosphorus", "hepatotoxicity", and "liver toxicity" through all relevant bibliographic databases, including Google Scholar, Scopus, Web of Science, PubMed, Medline, and Embase. The screening yielded more than 300 papers between the years 1977 and 2015. The obtained corpus was further sifted for the following search terms "acetylcholinesterase", "acetylcholine", "oxidative stress", "lipid peroxidation" "metabolic disorders", "mitochondrial toxicity", "genotoxicity", "histopathological", and "therapeutics" to narrow the choice of relevant articles to over 170, covering the last four decades of research.

\section{Histopathological evidence of OP hepatotoxicity}

Many studies confirm that the liver tissue is the primary target organ of OP toxicity (Figure 1).

Chlorpyrifos is a typical representative of the OPs, which causes detrimental effects both on liver function and 


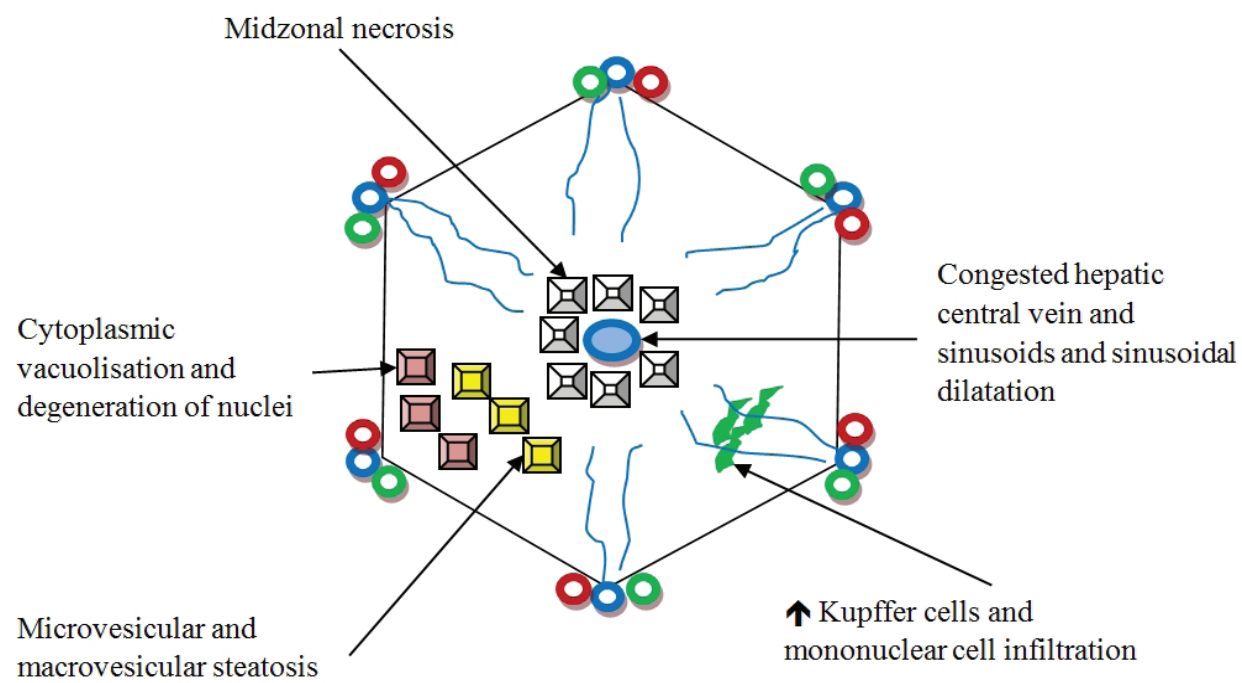

Figure 1 Histopathological disorders in the hepatic lobule after exposure to organophosphorus pesticides

structure. Almost forty years ago, Mikhail et al. (32) found that a two-day i.p. exposure to a sub-lethal dose of this insecticide resulted with mid-zonal liver necrosis, fatty deposition at the periphery, and glycogen deposition at one side of the hepatic cell and around the central vein. Goel et al. (33) studied liver histoarchitecture in chlorpyrifostreated rats and observed hepatocyte vacuolisation and necrosis, sinusoidal dilatation, and increase in binucleated cells at higher doses and longer exposure to chlorpyrifos. Recent findings by Ezzi et al. (34) suggest that chlorpyrifos had a dose-dependent effect on dilated sinusoids, central vein, and portal triad in rats.

An excessive amount of liver blood and degenerative changes were found in the liver of fish exposed to chlorpyrifos through contaminated water (35).

Another OP compound associated with liver damage in experimental animals is triazophos. Sharma et al. (36) observed a variety of histopathological findings, such as infiltration, vacuolisation, enlarged sinusoids, and necrosis in female albino rats exposed to triazophos.

Sub-chronic exposure of rats to methidathion caused mononuclear cell infiltration in all portal areas, sinusoidal dilatation, focal micro-vesicular steatosis, and parenchymal degenerations $(17,26)$.

Dose-dependent liver changes, including necrosis, cytoskeleton disarray, vacuolisation of the endothelial cells, damages in Disse's space, changes in nuclear shape, and heterochromatin distribution were evidenced in the liver of fish and rats exposed to sub-lethal doses of diazinon for a long time $(37,38)$.

Acute high dose of diazinon caused hypertrophy and swelling of hepatocytes, vacuolisation of cytoplasm, and macrovascular steatosis (39). Forty-minute inhalation of diazinon every other day in pregnant mice induced a dosedependent increase in the hepatocyte area, hepatocyte apoptosis, and a decrease in the sinusoid area of the foetal liver (40).
Malathion caused macrovesicular steatosis, apoptotic nuclei, granulovacuolar dystrophy lesions, and pericentrilobular vasodilatation in the liver of rats after about one month of low-dose exposure $(41,42)$. In contrast, Chakraborty et al. (43), reported only discrete to mild histological changes in the liver of rats exposed to a malathion dose about four times higher than in the study of Baconi et al. (41) for 15 days. A four-week daily oral exposure to sub-lethal doses of malathion caused hepatomegaly, necrotic lesions in the periportal lobules, cytoplasmic vacuolation around nuclei, and sinusoid expansion and atrophy of hepatocytes in rat liver $(44,45)$.

Parathion in the study by Chakraborty et al. (43), just like malathion, caused mild histopathological changes over 15 days of exposure. However, methyl parathion in the study by Undeger et al. (46) increased liver weight and caused hepatomegaly at lower doses than parathion. A sublethal dose of methyl parathion also caused cloudy swelling, bile stagnation, focal necrosis, atrophy, and vacuolisation in the liver tissue after contamination through water (47).

Dimethoate led to dose-dependent histological changes in rat liver, such as mononuclear cell infiltration, Kupffer cell count increase, congestion and dilatation of veins and sinusoids, necrosis, cytoplasmic vacuolisation, and degeneration of hepatocyte nuclei (22).

All of these studies investigated thion OPs that caused liver damage regardless of their structural differences. The exception is the study by Chakraborty et al. (43), but its comparison with Undeger et al. (46) may explain why. Namely, the presence of a bulky group in the side chain of OPs can make a great difference in the severity of liver injury.

As for oxon OPs, studies have reported hyalinisation, vacuolisation, nucleus necrosis, and hepatocellular oedema, and fatty degeneration in sub-acute exposure to low-dose of trichlorfon and acute exposure to omethoate $(48,49)$.

These disturbances in the morphological structure of the liver could be associated with a disruption of the tissue 
function, which could also be related to lower antioxidative capability, change in fatty and glycogen content in the liver, and the inhibition of some enzymes contributing to lipid, protein, and carbohydrate metabolism needed to preserve the integrity of the liver tissue.

\section{Biochemical evidence of OP hepatotoxicity}

Figure 2 shows some of the possible biochemical changes in the serum and liver after OP exposure. The most common serum biomarkers of liver damage are aspartate transaminase (AST) and alanine transaminase (ALT). There is strong evidence that both are increased by dimethoate, monocrotophos, methyl parathion, dichlorvos, fenitrothion, omethoate, chlorpyrifos, 2-butenoic acid-3(diethoxyphosphinothioyl) methyl ester (RPR-II), diazinon, and methidathion $(21,33,39,49-64)$. It is interesting to note that the increases in AST and ALT are often accompanied by increases in the inflammatory mediator tumour necrosis factor alpha (TNF- $\alpha)(39,49,51,65)$. In humans, higher AST and ALT were reported in tobacco farmworkers in India (66) and farm workers in Gadap Karachi, Pakistan exposed to a mixture of pesticides, including OPs (67).

Other biomarkers of liver damage include acid phosphatase (AcP) and alkaline phosphatase (ALP). Sub- lethal concentrations of methamidophos, phorate, and RPR-II seem to increase AcP and ALP in plasma and lower them in the liver tissue of animals $(60,68,69)$. Similar findings have been reported for sub-chronic monocrotophos, methyl parathion, and dimethoate effects on ALP and AcP levels in plasma, but not in the liver, where they also increased $(21,59)$.

Impaired bile flow and biliary excretion could also serve as indirect indicators of liver damage (70). Goel and Dhawan (71), for example, suggested that poor biliary excretion and longer half-life of $99 \mathrm{mTc}$-mebrofenin in chlorpyrifos-treated rats reflected impaired hepatobiliary function.

As impaired liver function affects the metabolism, consequently it also affects the concentrations of waste products. Several animal studies have pointed to the indirect effects of sub-lethal doses of phorate, fenitrothion, and dimethoate on urea and bilirubin $(58,64,72)$. In humans occupationally exposed to a wide range of OPs, blood urea nitrogen and albumin along with serum aminotransferases are frequently elevated $(30,73)$. Since, there are limited data about the hepatotoxicity of OPs in humans, it is not certain whether changes in biochemical parameters indicate actual liver damage.

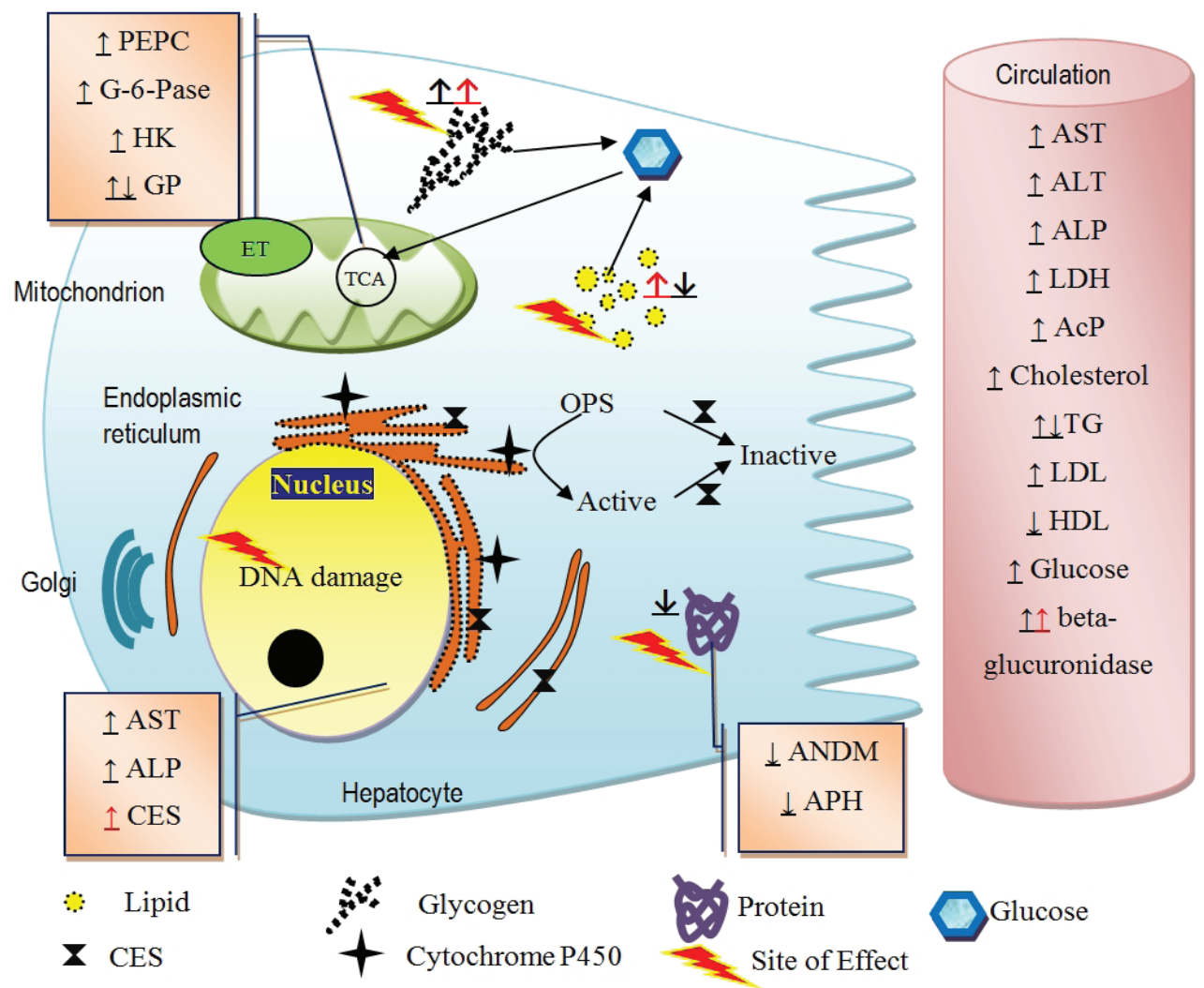

Figure 2 Possible disturbance pathways in the liver tissue after exposure to organophosphorus pesticides. Black arrows indicate long-term exposure and red ones acute poisoning.

Abbreviations: $A c P=$ acid phosphatase; $A L P=$ alkaline phosphatase; $A L T=$ alanine aminotransferase; $A N D M=$ aminopyrine- $N$ demethylase; $A P H=$ aniline-p-hydroxylase; $A S T=$ aspartate aminotransferase; $C E S=$ carboxylesterase $;$ G6Pase $=$ glucose-6phosphatase; $G P=$ glycogen phosphorylase; $H D L=$ high-densitylipoprotein; $H K=$ hexokinase; $L D L=$ low-density lipoprotein; $P E P C$ = phosphoenolpyruvate carboxykinase; $T G=$ triglyceride 


\section{Possible effects of OPs on hepatic carbohydrate, lipid, and protein metabolism}

Histological disorders are related to the metabolic capacity of the liver. Figure 2 illustrates how disturbances in the histoarchitecture of the liver induced by OPs can affect its performance in the enzymatic pathways involved in the metabolism of lipids, carbohydrates, and proteins in the cytoplasm, mitochondria, and peroxisomes (9). Animal studies suggest that the activity of glycogen phosphorylase (GP), the enzyme that breaks glycogen into glucose and reduces the hepatic glycogen content, increases after fenitrothion and malathion exposure $(74,75)$. This is supported by a drop in hepatic glycogen concentration after fenthion exposure in fish (76). In a study by Rezg et al. (44), however, sub-chronic exposure to malathion decreased GP activity by $50 \%$ and increased hexokinase activity by $10 \%$ but had no effect on blood glucose levels. Phosphoenolpyruvate carboxykinase (PEPCK) plays a crucial role in gluconeogenesis, and several studies have reported that malathion and diazinon increase the activity of both GP and PEPCK $(75,77-79)$. Two other hepatic gluconeogenesis enzymes, namely glucose-6-phosphatase and malate dehydrogenase, are affected by acute exposure to OPs, as reported for acephate (80). In contrast, Sharma et al. (23), found no significant changes in glucose-6phosphate dehydrogenase (G6PD) activity in acute exposure to dimethoate. In another study (81), hepatic malate dehydrogenase involved in the citric acid cycle and gluconeogenesis was downregulated in rats exposed to a sub-lethal dose of diazinon. OPs also seem to lower glucokinase, a liver enzyme contributing to glycogen synthesis, as reported for dichlorvos exposure (82). All of these studies indicated changes in carbohydrate metabolism, but their inconsistencies may be owed to the type of the investigated OP, route of administration, as well as animal species and strain.

On the other hand, diazinon-poisoned rats showed increased lactate production (83). This increase may be due to an increase in liver lactate dehydrogenase (LDH) activity, a key cytosolic enzyme in glycolysis, reported for the exposure to quinalphos, dimethoate, acephate, fenitrothion, and methidathion $(21,61,84-86)$. In a study by Mukhamedzhanov et al. (87) acute treatment of rats with intramuscular chlorophos and trichlorometaphos activated aldolase and LDH, which are both glycolytic enzymes. Higher hepatic LDH activity may represent a shift from mitochondrial respiration toward anaerobic glycolysis. Parallel changes in serum LDH and aminotransferases also suggest substantial liver involvement during exposure.

As a primary site of cellular energy generation and oxygen consumption, mitochondrion is a likely target for OPs, which may explain their non-cholinergic toxicity $(88$, 89). Recent research has focused on the possible roles of mitochondrial dysfunction in OP-induced toxicity through mitochondrial respiration rate, respiratory chain enzymes
(90-94), energy production (93, 95-97), and cell death (98100). Several studies have found an association between mitochondrial dynamics and malathion-induced drop in mitochondrial ATP synthesis in rat liver (9) or quinalphos and acephate effects on liver succinic dehydrogenase (84, $85,101)$ and ATPase activities (87) - all of them the key enzymes for oxidative phosphorylation. Two studies in rats $(90,93)$ point to an OP-induced drop in the liver mitochondrial respiratory control ratio (RCR), especially by dichlorvos. This decline may be related to changes in the inner mitochondrial membrane permeability caused by lipid peroxidation. A time-dependent decrease in carbonic anhydrase activity was reported in fish exposed to chlorpyrifos (35). Carbonic anhydrase in hepatocyte mitochondria plays a vital role in the regulation of ionic balance, which is required in metabolic reactions, production of ATP, and transport processes (102).

The effects of OPs, most notably diazinon and fenitrothion, on liver protein metabolism include the reduction of total protein, albumin, calcium-binding protein, and regucalcin (which is involved in $\mathrm{Ca}^{2+}$ transport) (51, 64, 72, 81, 86).

Diazinon can lower the liver activity of fumarylacetoacetase, which catalyses the hydrolysis of 4-fumarylacetacetate to acetoacetate and fumarate as part of the phenylalanine and tyrosine catabolism pathways and contributes to mental retardation (81). It can also affect carbamoyl-phosphate synthase, which catalyses the synthesis of carbamoyl phosphate from glutamine or ammonia and bicarbonate, and S-adenosylmethionine synthetase, which is vital for nucleic acid and protein synthesis (81).

One study revealed that methyl parathion affected the liver expression of chaperones and proteins regulating the cytoskeleton system, cell metabolism and signalling, electron transport, and hormone receptors in zebrafish (103).

Some studies reported OP-induced changes in liver lipid profiles. Nagaraju et al. (104) reported dyslipidaemia, including higher triglycerides and lower HDL-C in rats chronically exposed to monocrotophos. Sub-lethal fenitrothion reversibly increased triglycerides and cholesterols in mice after 90 days of exposure. All values returned to normal, except for triglycerides (72). Trichlorfon at the concentration of $2 \mathrm{mg} \mathrm{L}^{-1}$ decreased hepatic hormonesensitive lipase, very-low-density lipoprotein, and apolipoprotein B100 levels, which is associated with impaired lipid transport and accumulation of lipids in hepatocytes (105). However, low-dose OPs may not be contributing to lipid metabolism. In an in vitro study by Takeuchi et al. (106) reported that OPs at concentrations as low as $10^{-5} \mathrm{~mol} \mathrm{~L}^{-1}$ did not activate peroxisome proliferatoractivated receptors, which are ligand-dependent transcription factors and key regulators of lipid metabolism. 
Possible effects of OPs on hepatic oxidative stress pathways

Formation of reactive oxygen species (ROS) and reactive intermediates after exposure to pesticides can cause hepatotoxicity (107). Extensive data suggest that free radical formation and oxidative stress can be a major contributor to the toxicity of pesticides (108-113). OPs are oxidants and impair enzymatic antioxidant defences, including superoxide dismutase (SOD), catalase (CAT), glutathione peroxidase (GPx), and glutathione S-transferase (GST) (23, 34, 44, 114-129). Malathion, methyl parathion, and parathion in HepG2 cell lines, as well as chlorpyrifos, methidathion, chlorfenvinphos, and dimethoate in rat liver and diazinon in fish most probably disrupt membrane lipids through oxidative stress $(23,35,61,116,118,130-135)$. Table 1 summarises the key changes in antioxidant defence and oxidative stress biomarkers caused by OPs. Oxidative stress contributes to OP-induced toxicity through peroxidation of cellular macromolecules, which leads to a degradation of membrane phospholipids and proteins and cellular deterioration.
The main role in the induction of oxidative stress is played by the mitochondria. Leakage of electrons from the respiratory chain leads to the formation of ROS, triggers apoptotic pathways, and affects metabolism and ATP generation (136-138). Mitochondria are susceptible to oxidative damage, which manifests itself as changes in their transmembrane potential and weakened membrane integrity $(137,138)$. Mitochondrial oxidative damage is a major cause of many liver disorders, including chronic hepatitis, steatosis, ischemic injuries, aging, and inflammatory damage $(140,141)$. Several studies have evidenced the involvement of mitochondria in hepatotoxicity induced by OPs. For example, acute exposure to malathion and chronic exposure to dichlorvos and chlorpyrifos can increase the release of cytochrome $\mathrm{C}$ from mitochondria to cytosol and activate caspase- 3 by disrupting cellular antioxidant defences $(88,99,142)$. Diazinon can trigger apoptotic pathways by activating caspase- 9 and caspase- 3 , increase the $\mathrm{Bax} / \mathrm{Bcl}-2$ ratio and protein disulphide isomerase (with pro-apoptotic function), and suppress endoplasmic

Table 1 Summary of oxidative stress processes in the liver tissue triggered by organophosphorus pesticides

\begin{tabular}{|c|c|c|c|c|c|c|c|}
\hline $\begin{array}{l}\text { Pesticide } \\
\text { type }\end{array}$ & $\begin{array}{c}\text { Route of } \\
\text { administration }\end{array}$ & $\begin{array}{l}\text { Exposure } \\
\text { duration }\end{array}$ & Dose & Animal & $\begin{array}{c}\text { Antioxidant } \\
\text { enzymes }\end{array}$ & $\begin{array}{l}\text { Oxidative } \\
\text { stress } \\
\text { products }\end{array}$ & Antioxidants \\
\hline $\begin{array}{l}\text { Chlorpyrifos } \\
\text { (141) }\end{array}$ & gavage & $8 w$ & $\begin{array}{l}6.75 \mathrm{mg} \mathrm{kg}^{-1} \\
\text { per day }\end{array}$ & rat & - & $\begin{array}{c}\Delta \mathrm{LPO} \\
\Delta \mathrm{PC} \\
\Delta \mathrm{ROS}\end{array}$ & $\nabla \mathrm{TAC}$ \\
\hline $\begin{array}{l}\text { Chlorpyrifos } \\
\text { (121) }\end{array}$ & water & $\begin{array}{c}24,48, \& \\
96 \mathrm{~h}\end{array}$ & $\begin{array}{l}\text { single dose } \\
15.3 \text { and } \\
51 \mu \mathrm{g} \mathrm{L}^{-1}\end{array}$ & fish & $\begin{array}{l}\Delta \mathrm{SOD} \\
\nabla \mathrm{CAT}\end{array}$ & $\triangle \mathrm{LPO}$ & $\nabla \mathrm{TAC}$ \\
\hline $\begin{array}{l}\text { Chlorpyrifos } \\
(122)\end{array}$ & intramuscular & $7 \mathrm{~d}$ & $\begin{array}{l}\text { single dose } \\
50,100 \text {, and } \\
200 \mathrm{mg} \mathrm{kg}^{-1}\end{array}$ & rat & $\begin{array}{c}\nabla \mathrm{SOD}, \\
\nabla \mathrm{CAT} \\
\nabla \mathrm{G}_{6} \mathrm{PDH}, \\
\nabla \mathrm{GR}_{,} \Delta \mathrm{GPx}\end{array}$ & $\triangle \mathrm{LPO}$ & $\begin{array}{c}\boldsymbol{\nabla} \mathrm{GSH} \\
\boldsymbol{\Delta} \mathrm{GSSG}\end{array}$ \\
\hline $\begin{array}{l}\text { Chlorfen- } \\
\text { vinphos (133) }\end{array}$ & gavage & $14 \& 28 d$ & $\begin{array}{c}0.3 \mathrm{mg} \mathrm{kg}^{-1} \\
\text { per day }\end{array}$ & rat & - & $\Delta \mathrm{H}_{2} \mathrm{O}_{2}$ & $\nabla \mathrm{GSH}$ \\
\hline $\begin{array}{l}\text { Diazinon } \\
(134)\end{array}$ & water & 12 or $24 \mathrm{~h}$ & $\begin{array}{c}0.97, \\
1.95, \text { and } \\
3.95 \mathrm{mg} \mathrm{L}^{-1}\end{array}$ & fish & - & $\begin{array}{c}\Delta \mathrm{LPO} \\
\Delta \mathrm{PC}\end{array}$ & - \\
\hline Diazinon (39) & gavage & $7 \mathrm{~d}$ & $\begin{array}{l}\text { single dose } \\
335 \mathrm{mg} \mathrm{kg}^{-1}\end{array}$ & rat & $\begin{array}{l}\Delta \mathrm{SOD}, \\
\nabla \mathrm{MPO}\end{array}$ & - & - \\
\hline Diazinon (37) & water & $4 \mathrm{w}$ & $\begin{array}{c}0.1 \text { and } \\
0.2 \mathrm{mg} \mathrm{L}^{-1}\end{array}$ & fish & - & $\triangle \mathrm{LPO}$ & $\mathbf{\Delta} \mathrm{TAC}$ \\
\hline $\begin{array}{l}\text { Diazinon } \\
(123)\end{array}$ & drinking water & $1 \mathrm{w}$ & $10 \mathrm{mg} \mathrm{kg}^{-1}$ & rat & $\begin{array}{l}\nabla \mathrm{GPx}, \\
\nabla \mathrm{GST}, \\
\nabla \mathrm{CAT}\end{array}$ & - & $\nabla \mathrm{GSH}$ \\
\hline $\begin{array}{l}\text { Diazinon } \\
(124)\end{array}$ & water & $\begin{array}{l}1,7,15 \\
\& 30 \mathrm{~d}\end{array}$ & $\begin{array}{l}0.1,1, \text { and } \\
2 \mathrm{mg} \mathrm{L}^{-1}\end{array}$ & fish & $\Delta \mathrm{GST}$ & $\Delta \mathrm{LPO}$ & $\mathbf{\Delta} \mathrm{GSH}$ \\
\hline $\begin{array}{l}\text { Diaxinon } \\
(125)\end{array}$ & water & $\begin{array}{c}5,15, \& \\
30 \mathrm{~d}\end{array}$ & $\begin{array}{c}0.0036, \\
0.018, \text { and } \\
0.036 \mu \mathrm{g} \mathrm{L}^{-1}\end{array}$ & fish & $\begin{array}{l}\mathbf{\Delta} \mathrm{SOD} \\
\boldsymbol{\Delta} \mathrm{GPx} \\
\mathbf{\Delta} \mathrm{CAT}\end{array}$ & NC LPO & - \\
\hline $\begin{array}{l}\text { Dichlorvos } \\
(126)\end{array}$ & gavage & $3 \mathrm{w}$ & $\begin{array}{c}0.64, \\
1.60, \text { and } \\
4.00 \mathrm{mg} \mathrm{kg}^{-1} \\
\text { per day }\end{array}$ & rat & $\begin{array}{l}\Delta \mathrm{SOD} \\
\Delta \mathrm{CAT}\end{array}$ & $\begin{array}{c}\Delta \mathrm{LPO} \\
\Delta \mathrm{PC}\end{array}$ & - \\
\hline
\end{tabular}


Table 1 continued

\begin{tabular}{|c|c|c|c|c|c|c|c|}
\hline $\begin{array}{l}\text { Pesticide } \\
\text { type }\end{array}$ & $\begin{array}{c}\text { Route of } \\
\text { administration }\end{array}$ & $\begin{array}{l}\text { Exposure } \\
\text { duration }\end{array}$ & Dose & Animal & $\begin{array}{c}\text { Antioxidant } \\
\text { enzymes }\end{array}$ & $\begin{array}{l}\text { Oxidative } \\
\text { stress } \\
\text { products } \\
\end{array}$ & Antioxidants \\
\hline $\begin{array}{l}\text { Dimethoate } \\
\text { (118) }\end{array}$ & gavage & $30 \mathrm{~d}$ & $\begin{array}{c}0.6,6 \text { and } \\
30 \mathrm{mg} \mathrm{kg}^{-1} \\
\text { per day }\end{array}$ & rat & $\boldsymbol{\nabla}$ GST & $\Delta \mathrm{LPO}$ & $\boldsymbol{\nabla} \mathrm{GSH}$ \\
\hline $\begin{array}{l}\text { Dimethoate } \\
(21)\end{array}$ & gavage & $8 w$ & $\begin{array}{l}20 \mathrm{mg} \mathrm{kg}^{-1} \\
\text { per day }\end{array}$ & rat & $\begin{array}{l}\Delta \mathrm{SOD} \\
\Delta \mathrm{GPx} \\
\nabla \mathrm{CAT}\end{array}$ & $\Delta \mathrm{LPO}$ & - \\
\hline $\begin{array}{l}\text { Dimethoate } \\
\text { (23) }\end{array}$ & gavage & $24 \mathrm{~h}$ & $\begin{array}{l}\text { single dose } \\
45,75 \text {, and } \\
90 \mathrm{mg} \mathrm{kg}^{-1}\end{array}$ & rat & $\begin{array}{c}\Delta \mathrm{SOD} \\
\boldsymbol{\Delta} \mathrm{GPx} \\
\boldsymbol{\Delta} \mathrm{CAT}, \boldsymbol{\Delta} \mathrm{GR}\end{array}$ & $\Delta \mathrm{LPO}$ & $\boldsymbol{\nabla} \mathrm{GSH}$ \\
\hline $\begin{array}{l}\text { Dimethoate } \\
(127)\end{array}$ & intraperitoneal & $1 \mathrm{w}$ & $\begin{array}{c}1,5,10 \\
15 \text { and } \\
30 \mathrm{mg} \mathrm{kg}^{-1}\end{array}$ & rat & $\Delta \mathrm{CAT}$ & $\begin{array}{c}\Delta \mathrm{LPO} \\
\Delta \mathrm{PC}\end{array}$ & - \\
\hline $\begin{array}{l}\text { Fenitrothion } \\
(128)\end{array}$ & water & $1 \mathrm{w}$ & $0.04 \mathrm{mg} \mathrm{L}^{-1}$ & fish & $\begin{array}{l}\Delta \mathrm{SOD}, \\
\Delta \mathrm{CAT} \\
\Delta \mathrm{GST}\end{array}$ & - & - \\
\hline $\begin{array}{l}\text { Malathion } \\
\text { (129) }\end{array}$ & gavage & $4 \mathrm{w}$ & $\begin{array}{l}200 \mathrm{mg} \mathrm{kg}^{-1} \\
\text { per day }\end{array}$ & rat & - & $\Delta \mathrm{LPO}$ & $\begin{array}{l}\boldsymbol{\nabla} \mathrm{GSH}, \\
\boldsymbol{\nabla} \mathrm{TAC}\end{array}$ \\
\hline $\begin{array}{l}\text { Malathion } \\
\text { (45) }\end{array}$ & water & $4 \mathrm{w}$ & $\begin{array}{l}150 \mathrm{mg} \mathrm{kg}^{-1} \\
\text { per day }\end{array}$ & rat & $\begin{array}{l}\boldsymbol{\nabla} \text { SOD, } \boldsymbol{\nabla} \\
\text { CAT }\end{array}$ & $\Delta \mathrm{LPO}$ & $\boldsymbol{\nabla} \mathrm{GSH}$ \\
\hline $\begin{array}{l}\text { Malathion } \\
\text { (116) }\end{array}$ & drinking water & $4 \mathrm{w}$ & $\begin{array}{c}100,316, \\
1000 \text { and, } \\
1500 \mathrm{mg} \mathrm{L}^{-1}\end{array}$ & rat & $\begin{array}{l}\boldsymbol{\Delta} \mathrm{SOD} \\
\boldsymbol{\Delta} \mathrm{CAT}\end{array}$ & $\Delta \mathrm{LPO}$ & - \\
\hline $\begin{array}{l}\text { Methidathion } \\
(17,26,61)\end{array}$ & gavage & $24 \mathrm{~h}$ & $\begin{array}{l}\text { single dose } \\
8 \mathrm{mg} \mathrm{kg}^{-1}\end{array}$ & rat & - & $\Delta \mathrm{LPO}$ & - \\
\hline $\begin{array}{l}\text { Trichlorfon } \\
\text { (48) }\end{array}$ & water & $4 w$ & $\begin{array}{l}\text { 0.01, 0.1, } \\
1.0 \mathrm{mg} \mathrm{L}^{-1}\end{array}$ & frog & $\begin{array}{l}\Delta \mathrm{SOD}, \\
\Delta \mathrm{CAT}\end{array}$ & $\boldsymbol{\nabla}$ LPO & - \\
\hline $\begin{array}{l}\text { Triazophos } \\
\text { (36) }\end{array}$ & gavage & $4 \mathrm{w}$ & $\begin{array}{l}8.2,4.1, \text { and } \\
2.05 \mathrm{mg} \mathrm{kg}^{-1}\end{array}$ & rat & $\begin{array}{c}\boldsymbol{\nabla} \text { SOD, } \\
\boldsymbol{\nabla} \text { GPx, } \\
\boldsymbol{\nabla} \text { CAT, } \boldsymbol{\nabla} \text { GR, } \\
\nabla \text { GST }\end{array}$ & $\Delta \mathrm{LPO}$ & - \\
\hline
\end{tabular}

Abbreviations: $C A T=$ catalase; $G_{6} P D H=$ glucose-6-phosphate dehydrogenase; GPx = glutathione peroxidase; $G R=$ glutathione reductase; $G S H=$ reduced glutathione; $G S S G=$ oxidized glutathione; $G S T=$ glutathione $S$-transferase; $\mathrm{H}_{2} \mathrm{O}_{2}=$ hydrogen peroxide; $L P O=$ lipid peroxidation; $M P O=$ myeloperoxidase $; C=$ no change; $P C=$ protein carbonyl; $S O D=$ superoxide dismutase; $T A C=$ total antioxidant capacity

chaperone (with anti-apoptotic properties) in the liver (81, 143).

\section{Possible effect of OPS on hepatic biotransformation}

Animal studies of OP effects on important cytochromes and microsomal enzymes that affect biotransformation of compounds and drug metabolism report controversial results. Several studies reported chlorpyrifos, malathion, and parathion as potent inhibitors of cytochrome $\mathrm{P} 450$ (CYP) 3A and 2C11, cytochrome b5, and aminopyrine $\mathrm{N}$-demethylase (ANDM) $(42,144,121)$, but some reported no effect (parathion on CYP 2A1, 2A2, and 2C6) (144) or an increase (iprobenfos on CYP 1A mRNA transcription) (143). Low-dose dimethoate administered for two weeks increased the mRNA expression of CYP 2D1 and higher metabolism of metoprolol in rats (145). Parathion and paraoxon decreased the activity of rat liver benzo(a)pyrene hydroxylase, which is governed by CYP 1A1 (146).

In humans, Van der Meer et al. (147) reported complete inhibition of atropine metabolism in OP-poisoned patients and that this was owed to the inhibition of hepatic microsomal enzymes.

Several in vitro studies have also indicated OP effects on aryl hydrocarbon receptor (AhR), a ligand-dependent transcription factor that regulates genes involved in xenobiotic metabolism (148-150).

Possible mutagenic, genotoxic, and carcinogenic effects of OPs

Mutagenic and genotoxic effects of pesticides have been evidenced by a variety of tests showing gene mutations, chromosomal aberrations, and micronucleus formation (151-154). The mutagenic effects of OPs are owed to the direct alkylating ability of the parental molecule and its metabolites (130, 131, 155).

The genotoxic potential of pesticides has been demonstrated by the comet assay in exposed workers in India and Iran $(156,157)$. Several in vivo and in vitro studies reported that the genotoxicity of OPs such as dimethoate, methyl parathion, chlorpyrifos, phorate, and malathion in 
hepatocytes was associated with oxidative damage (130, 131, 127, 149-159). A study by Hreljac et al. (160) showed that methyl paraoxon was less genotoxic in HepG2 cells than its parent OP methyl parathion. This suggests that the genotoxicity of methyl parathion and methyl paraoxon is AChE-independent and that other mechanisms are involved in this process. In the same study, on the other hand, dimefox, a highly toxic OP, did not induce DNA strand breaks but showed mitogenic activity.

As for carcinogenicity, Reuber (161) reports that dimethoate and omethoate, its toxic metabolite, can cause benign and malignant neoplasms in the liver. However, the International Agency for Research on Cancer (IARC) could not find enough evidence to classify dimethoate as potential carcinogen (162). Furthermore, a study by Bonner et al. (163) did not associate malathion with cancer in pesticide applicators. All these findings suggest that the agents causing low DNA damage are generally not mutagenic or carcinogenic, and those causing sustained DNA and cell damage (but not cell death) are mutagenic and/or carcinogenic (164).

\section{Conclusions and recommendations to control OP- induced liver damage}

This literature review abundantly evidences that OPs can have significant and harmful effects on the liver and points to the need of regular liver function monitoring in long-term occupational and short-term accidental exposure to OP insecticides.

The presented research (summarised in the supplementary Table 2 at the end of the review) suggests that the key mechanisms of OP action are disturbances in the liver metabolism and mitochondrial metabolic pathways caused by oxidative damage. This conclusion is reinforced by other OP effects, such as apoptosis, cell toxicity, genotoxicity, and tissue damage induced by ROS triggered by OP exposure.

The best course of action to counter OP liver and mitochondrial toxicity in acute, subacute, sub-chronic, or chronic exposure is therefore to use antioxidants $(89,119$, $165,166)$. The choice of antioxidants is wide, but several have been evidenced as potent against OP toxicity, including selenium (167), $N$-acetylcysteine (NAC), pentoxifylline (PTX), and alpha-tocopherol. NAC has been reported to significantly decrease lipid peroxidation, hospital time, and mortality in poisoned patients (168), whereas PTX and alpha-tocopherol reverse OP-induced effects on glutathione, nitrotyrosine, CAT, and GPx $(65,169,170)$. Recently, a new generation of possible antidotes to OP poisoning has raised interests, the so called OP hydrolases produced by bacteria, but research has not yet reached clinical stage (171).

Since few studies have compared the effects of OPs with different structures, little is known about the exact relationship between the severity and mechanism of liver damage and the chemical structure of these toxins.

We hope that future in vitro, in vivo, and clinical trials will answer the remaining questions about the mechanisms of OP hepatotoxicity and its management.

\section{Conflict of interest}

None to declare.

\section{Funding}

The authors received no financial support for the research done in the preparation of this article.

Table 2 (supplementary) Summary of the key hepatotoxic effects by organophosphorus pesticides

\begin{tabular}{|c|c|c|c|}
\hline Name & Structure & $\begin{array}{l}{ }^{1} \text { Pesticide type } \\
{ }^{2} \text { Chemical formula } \\
{ }^{3} \text { CAS number }\end{array}$ & Hepatotoxic effects \\
\hline $\begin{array}{l}\text { Chlorpyrifos } \\
(33,34,35,53, \\
71,132,141,121, \\
158,122)\end{array}$ & & $\begin{array}{l}{ }^{1} \text { Insecticide } \\
{ }^{2} \mathrm{C}_{9} \mathrm{H}_{11} \mathrm{Cl}_{3} \mathrm{NO}_{3} \mathrm{PS} \\
{ }^{3} 2921-88-2\end{array}$ & $\begin{array}{c}\text { - Histopathological changes (rat and fish) } \\
\text { - Changes in hepatobiliary system (rat) } \\
\text { - Changes in serum liver damage biomarkers } \\
\text { (rat) } \\
\text { - Downregulation of CYP 3A (fish) } \\
\text { - Oxidative stress induction (rat and fish) } \\
\text { - DNA damages (rat) } \\
\text { - Apoptosis induction (rat) }\end{array}$ \\
\hline Triazophos (36) & & $\begin{array}{c}{ }^{1} \text { Acaricide, insecticide, and } \\
\text { nematicide } \\
{ }^{2} \mathrm{C}_{12} \mathrm{H}_{16} \mathrm{~N}_{3} \mathrm{O}_{3} \mathrm{PS} \\
{ }^{3} 24017-47-8\end{array}$ & $\begin{array}{l}\text { - Histopathological changes (rat) } \\
\text { - Oxidative stress induction (rat) }\end{array}$ \\
\hline $\begin{array}{l}\text { Methidathion }(17, \\
26,61)\end{array}$ & & $\begin{array}{c}{ }^{1} \text { Acaricide and insecticide } \\
{ }^{2} \mathrm{C}_{6} \mathrm{H}_{11} \mathrm{~N}_{2} \mathrm{O}_{4} \mathrm{PS}_{3} \\
{ }^{3} 950-37-8\end{array}$ & $\begin{array}{l}\text { - Histopathological changes (rat) } \\
\text { - Oxidative stress induction (rat) }\end{array}$ \\
\hline
\end{tabular}




\begin{tabular}{|c|c|c|c|}
\hline Name & Structure & $\begin{array}{l}{ }^{1} \text { Pesticide type } \\
{ }^{2} \text { Chemical formula } \\
{ }^{3} \text { CAS number }\end{array}$ & Hepatotoxic effects \\
\hline $\begin{array}{l}\text { Diazinon }(13, \\
37-40,51,63,78, \\
81,83,154,167 \text {, } \\
172-125)\end{array}$ & & $\begin{array}{c}{ }^{1} \text { Insecticide } \\
{ }^{2} \mathrm{C}_{12} \mathrm{H}_{21} \mathrm{~N}_{2} \mathrm{O}_{3} \mathrm{PS} \\
{ }_{3}^{3} 333-41-5\end{array}$ & $\begin{array}{l}\text { - Histopathological changes (rat and fish) } \\
\text { - Changes in serum liver damage biomarkers } \\
\text { (rat) } \\
\text { - Glucose, lipid, and protein metabolism (rat) } \\
\text { - Oxidative stress induction (rat and fish) } \\
\text { - DNA damages (rat) } \\
\text { - Apoptosis induction (rat) }\end{array}$ \\
\hline $\begin{array}{l}\text { Malathion }(12,20 \text {, } \\
25,41-45,75-77 \text {, } \\
94,95,116,169 \text {, } \\
129)\end{array}$ & & $\begin{array}{l}{ }^{1} \text { Insecticide } \\
{ }^{2} \mathrm{C}_{10} \mathrm{H}_{19} \mathrm{O}_{6} \mathrm{PS}_{2} \\
{ }^{3} 121-75-5\end{array}$ & $\begin{array}{c}\text { - Histopathological changes (rat) } \\
\text { - Changes in serum liver damage biomarkers } \\
\text { (rat) } \\
\text { - Glucose, lipid, and protein metabolism (rat) } \\
\text { - Oxidative stress induction (rat) } \\
\text { - Metabolism (chicken) } \\
\text { - Apoptosis induction (rat) }\end{array}$ \\
\hline
\end{tabular}

- Histopathological changes (rat)

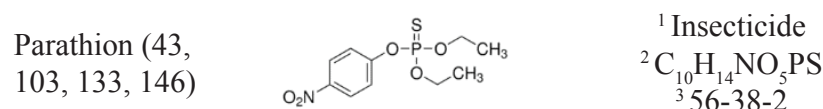

$\mathrm{C}_{10} \mathrm{H}_{14} \mathrm{NO}_{5} \mathrm{PS}$

${ }^{3} 56-38-2$
- Changes in serum liver damage biomarkers (rat)

- Downregulation of CYP 3A and 2C11 (fish)

- Lipid metabolism (fish)

- Oxidative stress induction (rat)

- Decrease in benzo(a)pyrene metabolism (rat) - Apoptosis induction (rat)

- Histopathological changes and hepatomegaly (rat)

- Changes in serum liver damage biomarkers (rat)

- Lipid metabolism (fish)
Dimethoate (21$23,58,60,118$, $145,127)$

$$
\mathrm{H}_{3} \mathrm{C}^{-\mathrm{N}} \prod_{\mathrm{O}}^{\substack{\mathrm{S}-\mathrm{P}-\mathrm{OCH}_{3} \\ \mathrm{OCH}_{3}}}
$$

${ }^{1}$ Acaricide and insecticide

$$
{ }^{2} \mathrm{C}_{5} \mathrm{H}_{12} \mathrm{NO}_{3} \mathrm{PS}_{2}
$$$$
{ }^{3} \text { 60-51-5 }
$$

- Histopathological changes (rat)

- Changes in serum liver damage biomarkers (rat)

- Upregulation of CYP 2D1 (rat)

- Cause benign and malignant neoplasm (rat) - Oxidative stress induction (rat)

\begin{tabular}{|c|c|c|c|}
\hline $\begin{array}{l}\text { Omethoate (49, } \\
161)\end{array}$ & 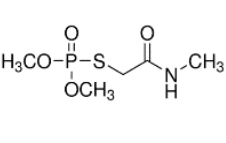 & $\begin{array}{l}{ }^{1} \text { Acaricide and insecticide } \\
{ }^{2} \mathrm{C}_{5} \mathrm{H}_{12} \mathrm{NO}_{4} \mathrm{PS} \\
{ }^{3} 1113-02-6\end{array}$ & $\begin{array}{l}\text { - Histopathological changes (rat) } \\
\text { - Changes in serum liver damage biomarkers } \\
\text { (rat) } \\
\text { - Cause benign and malignant neoplasm (rat) }\end{array}$ \\
\hline $\begin{array}{l}\text { Trichlorfon } \\
(48,105,115)\end{array}$ & $\begin{array}{l}\mathrm{OH} \\
\mathrm{O} \\
\mathrm{P} \\
\mathrm{P}\end{array} \mathrm{CCl}_{3}$ & $\begin{array}{l}{ }^{1} \text { Insecticide } \\
{ }^{2} \mathrm{C}_{4} \mathrm{H}_{8} \mathrm{Cl}_{3} \mathrm{O}_{4} \mathrm{P} \\
{ }_{3}^{3} 52-68-6\end{array}$ & $\begin{array}{l}\text { - Histopathological changes (frog) } \\
\text { - Lipid metabolism (fish) } \\
\text { - Oxidative stress induction (rat and frog) }\end{array}$ \\
\hline $\begin{array}{l}\text { Monocrotophos } \\
(59,65,104)\end{array}$ & & $\begin{array}{l}{ }^{1} \text { Insecticide } \\
{ }^{2} \mathrm{C}_{7} \mathrm{H}_{14} \mathrm{NO}_{5} \mathrm{P} \\
{ }^{3} 6923-22-4\end{array}$ & $\begin{array}{c}\text { - Changes in serum liver damage biomarkers } \\
\text { (rat) } \\
\text { - Glucose and lipid metabolism (rat) }\end{array}$ \\
\hline $\begin{array}{l}\text { Dichlorvos }(82, \\
88,93,126)\end{array}$ & & $\begin{array}{l}{ }^{1} \text { Insecticide } \\
{ }^{2} \mathrm{C}_{4} \mathrm{H}_{7} \mathrm{Cl}_{2} \mathrm{O}_{4} \mathrm{P} \\
{ }^{3} 62-73-7\end{array}$ & $\begin{array}{c}\text { - Histopathological changes (rat) } \\
\text { - Changes in serum liver damage biomarkers } \\
\text { (rat) } \\
\text { - Glucose and lipid metabolism (rat) } \\
\text { - Oxidative stress induction (rat) } \\
\text { - Apoptosis induction (rat) }\end{array}$ \\
\hline $\begin{array}{l}\text { Fenitrothion }(54, \\
64,72,74,86,128)\end{array}$ & $\mathrm{O}_{2} \mathrm{~N}$ & $\begin{array}{l}{ }^{1} \text { Insecticide } \\
{ }^{2} \mathrm{C}_{9} \mathrm{H}_{12} \mathrm{NO}_{5} \mathrm{PS} \\
{ }^{3} 122-14-5\end{array}$ & $\begin{array}{l}\text { - Changes in serum liver damage biomarkers } \\
\text { (rat) } \\
\text { - Glucose and lipid metabolism (fish) } \\
\text { - Oxidative stress induction (rat) }\end{array}$ \\
\hline
\end{tabular}

- Histopathological changes (rat) 


\begin{tabular}{|c|c|c|c|}
\hline Name & Structure & $\begin{array}{l}{ }^{1} \text { Pesticide type } \\
{ }^{2} \text { Chemical formula } \\
{ }^{3} \text { CAS number }\end{array}$ & Hepatotoxic effects \\
\hline RPR-II (60) & 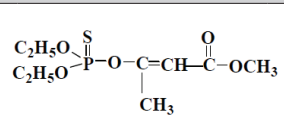 & $\begin{array}{l}{ }^{1} \text { Insecticide } \\
{ }^{2} \mathrm{C}_{6} \mathrm{H}_{10} \mathrm{O}_{2} \\
{ }^{3} 924-50-5\end{array}$ & $\begin{array}{l}\text { - Changes in serum liver damage biomarkers } \\
\text { (fish) }\end{array}$ \\
\hline $\begin{array}{l}\text { Methamidophos } \\
\text { (69) }\end{array}$ & $\begin{array}{l}\stackrel{\mathrm{O}}{\mathrm{M}} \\
\mathrm{H}_{3} \mathrm{CO}-\mathrm{PH}_{1}-\mathrm{NH}_{2} \\
\stackrel{\mathrm{S}}{\mathrm{S}} \mathrm{H}_{3}\end{array}$ & $\begin{array}{l}{ }^{1} \text { Insecticide } \\
{ }^{2} \mathrm{C}_{2} \mathrm{H}_{8} \mathrm{NO}_{2} \mathrm{PS} \\
{ }^{3} 10265-92-6\end{array}$ & $\begin{array}{l}\text { - Changes in serum liver damage biomarkers } \\
\text { (rat) } \\
\text { - Histopathological changes (rat) } \\
\text { - Oxidative stress induction (rat) }\end{array}$ \\
\hline Phorate $(68,159)$ & & $\begin{array}{l}{ }^{1} \text { Acaricide and insecticide } \\
{ }^{2} \mathrm{C}_{7} \mathrm{H}_{17} \mathrm{O}_{2} \mathrm{PS}_{3} \\
{ }^{3} 298-02-2\end{array}$ & $\begin{array}{l}\text { - Changes in serum liver damage biomarkers } \\
\text { (rat) } \\
\text { - DNA damages (rat) } \\
\text { - Oxidative stress induction (rat) }\end{array}$ \\
\hline Fenthion $(76,133)$ & & $\begin{array}{l}{ }^{1} \text { Acaricide, avicide, } \\
\text { and insecticide } \\
{ }^{2} \mathrm{C}_{10} \mathrm{H}_{15} \mathrm{O}_{3} \mathrm{PS}_{2} \\
{ }^{3} 55-38-9\end{array}$ & $\begin{array}{l}\text { - Glucose metabolism (fish) } \\
\text { - Oxidative stress induction (fish) }\end{array}$ \\
\hline Acephate $(80,84)$ & & $\begin{array}{l}{ }^{1} \text { Insecticide } \\
{ }^{2} \mathrm{C}_{4} \mathrm{H}_{10} \mathrm{NO}_{3} \mathrm{PS} \\
{ }^{3} 30560-19-1 \\
\end{array}$ & $\begin{array}{c}\text { - Changes in serum liver damage biomarkers } \\
\text { (rat) } \\
\text { - Glucose metabolism (rat) }\end{array}$ \\
\hline Chlorophos (87) & & $\begin{array}{c}{ }^{1} \text { Insecticide and vermicide } \\
{ }^{2} \mathrm{C}_{4} \mathrm{H}_{8} \mathrm{Cl}_{3} \mathrm{O}_{4} \mathrm{P} \\
{ }^{3} 52-68-6\end{array}$ & $\begin{array}{c}\text { - Changes in serum liver damage biomarkers } \\
\text { (rat) } \\
\text { - Glucose metabolism (rat) }\end{array}$ \\
\hline $\begin{array}{l}\text { Trichlorometaphos } \\
\text { (87) }\end{array}$ & & $\begin{array}{l}{ }^{1} \text { Insecticide } \\
{ }^{2} \mathrm{C}_{9} \mathrm{H}_{10} \mathrm{Cl}_{3} \mathrm{O}_{3} \mathrm{PS} \\
{ }^{3} 2633-54-7\end{array}$ & $\begin{array}{c}\text { - Changes in serum liver damage biomarkers } \\
\text { (rat) } \\
\text { - Glucose metabolism (rat) }\end{array}$ \\
\hline Quinalphos (85) & $\mathrm{O} \sim \mathrm{CH}_{3}$ & $\begin{array}{l}{ }^{1} \text { Insecticide } \\
{ }^{2} \mathrm{C}_{12} \mathrm{H}_{15} \mathrm{~N}_{2} \mathrm{O}_{3} \mathrm{PS} \\
{ }^{3} 13593-03-8\end{array}$ & - Glucose metabolism (fish) \\
\hline
\end{tabular}

\section{REFERENCES}

1. Galloway T, Handy R. Immunotoxicity of organophosphorous pesticides. Ecotoxicology 2003;12:345-63. PMID: 12739880

2. Costa LG. Current issues in organophosphate toxicology. Clin Chim Acta 2006;366:1-13. doi: 10.1016/j. cca.2005.10.008

3. Ghazala, Mahboob S, Ahmad L, Sultana S, Alghanim K, Al-Misned F, Ahmad Z. Fish cholinesterases as biomarkers of sublethal effects of organophosphorus and carbamates in tissues of Labeo Rohita. J Biochem Mol Toxicol 2013;28:13742. doi: $10.1002 / \mathrm{jbt} .21545$

4. Shadnia S, Okazi A, Akhlaghi N, Sasanian G, Abdollahi M. Prognostic value of long QT interval in acute and severe organophosphate poisoning. J Med Toxicol 2009;5:196-9. PMCID: PMC3550412

5. Gunnell D, Eddleston M, Phillips MR, Konradsen F. The global distribution of fatal pesticide self-poisoning: systematic review. BMC Public Health 2007;7:357. doi: 10.1186/1471-2458-7-357

6. Jeyaratnam J. Acute pesticide poisoning: a major global health problem. World Health Stat Q 1990;43:139-44. PMID: 2238694
7. Soltaninejad K, Abdollahi M. Current opinion on the science of organophosphate pesticides and toxic stress: a systematic review. Med Sci Monit 2009;15:RA75-90. PMID: 19247260

8. Dettbern WD, Milatovic D, Gupta RC. Toxicology of organophosphate and carbamate compounds. In: Gupta RC, editor. Oxidative stress in anticholinesterase-induced excitotoxicity. London: Academic Press; 2006. p. 511-29.

9. Karami-Mohajeri S, Abdollahi M. Toxic influence of organophosphate, carbamate, and organochlorine pesticides on cellular metabolism of lipids, proteins, and carbohydrates: A systematic review. Hum Exp Toxicol 2011;30:1119-40. doi: 10.1177/0960327110388959

10. Kumar S, Fareedullah M, Sudhakar Y, Venkateswarlu B, Kumar E. Current review on organophosphorus poisoning. Arch Appl Sci Res 2010;2:199-215 [displayed 20 November 2017]. Available at https://pdfs.semanticscholar. org/38c2/61960db82980befd9c87485551e2280991f5.pdf

11. Hoekstra LT, de Graaf W, Nibourg GA, Heger M, Bennink RJ, Stieger B, van Gulik TM. Physiological and biochemical basis of clinical liver function tests: a review. Ann Surg 2013;257:27-36. doi: 10.1097/SLA.0b013e31825d5d47

12. Lasram MM, Annabi AB, Elj NE, Selmi S, Kamoun A, ElFazaa S, Gharbi N. Metabolic disorders of acute exposure to malathion in adult Wistar rats. J Hazard Mater 2009;163:10525. doi: 10.1016/j.jhazmat.2008.07.059 
13. Kalender S, Ogutcu A, Uzunhisarcikli M, Acikgoz F, Durak D, Ulusoy Y, Kalender Y. Diazinon-induced hepatotoxicity and protective effect of vitamin $\mathrm{E}$ on some biochemical indices and ultrastructural changes. Toxicology 2005;211:197206. doi: 10.1016/j.tox.2005.03.007

14. Chan JY, Chan SH, Dai KY, Cheng HL, Chou JL, Chang AY. Cholinergic-receptor-independent dysfunction of mitochondrial respiratory chain enzymes, reduced mitochondrial transmembrane potential and ATP depletion underlie necrotic cell death induced by the organophosphate poison mevinphos. Neuropharmacology 2006;51:1109-19. doi: 10.1016/j.neuropharm.2006.06.024

15. Astiz M, de Alaniz MJ, Marra CA. Effect of pesticides on cell survival in liver and brain rat tissues. Ecotoxicol Environ Saf 2009;72:2025-32. doi: 10.1016/j.ecoenv.2009.05.001

16. Satar S, Satar D, Tap O, Koseoglu Z, Kaya M. Ultrastructural changes in rat liver treated with pralidoxime following acute organophosphate poisoning. Mt Sinai J Med 2004;71:405-10. PMID: 15592660

17. Gokalp O, Gulle K, Sulak O, Cicek E, Altuntas I. The effects of methidathion on liver: role of vitamins $\mathrm{E}$ and $\mathrm{C}$. Toxicol Ind Health 2003; 19:63-7. doi: 10.1191/0748233703th176oa

18. Hettwer H. Histochemische Untersuchungen an Leber und Niere der Ratte nach Phosphorsäureestervergiftungen [Histochemical investigations on liver and kidney of the rat after intoxication with organophosphates (author's transl), in German]. Acta Histochem 1975;52:239-52. PMID: 171902

19. Hettwer H, Gericke C. Lipide der Plasmamembranen und der Mitochondrien aus Rattenleber nach ParaoxonIntoxikation [Lipids of plasmamembranes and of mitochondria in rat liver after intoxication with paraoxon (author's transl), in German]. Arch Toxicol 1977;38:251-60. PMID: 579971

20. Delgado E, Streck E, Quevedo J, Dal-Pizzol F. Mitochondrial respiratory dysfunction and oxidative stress after chronic malathion exposure. Neurochem Res 2006;31:1021-5. doi: 10.1007/s11064-006-9111-1

21. Saafi EB, Louedi M, Elfeki A, Zakhama A, Najjar MF, Hammami M, Achour L. Protective effect of date palm fruit extract (Phoenix dactylifera L.) on dimethoate inducedoxidative stress in rat liver. Exp Toxicol Pathol 2011;63:43341. doi: $10.1016 /$ j.etp.2010.03.002

22. Sayim F. Dimethoate-induced biochemical and histopathological changes in the liver of rats. Exp Toxicol Pathol 2007;59:237-43. doi: 10.1016/j.etp.2007.05.008

23. Sharma Y, Bashir S, Irshad M, Gupta SD, Dogra TD. Effects of acute dimethoate administration on antioxidant status of liver and brain of experimental rats. Toxicology 2005;206:4957. doi: 10.1016/j.tox.2004.06.062

24. Maliwal BP, Guthrie FE. Interaction of insecticides with human plasma lipoproteins. Chem Biol Interact 1981;35:17788. doi: /10.1016/0009-2797(81)90141-1

25. Al-Attar AM. Physiological and histopathological investigations on the effects of alpha-lipoic acid in rats exposed to malathion. J Biomed Biotechnol 2010;2010:203503. doi: 10.1155/2010/203503

26. Sutcu R, Altuntas I, Yildirim B, Karahan N, Demirin H, Delibas N. The effects of subchronic methidathion toxicity on rat liver: role of antioxidant vitamins $\mathrm{C}$ and $\mathrm{E}$. Cell Biol Toxicol 2006;22:221-7. doi: 10.1007/s10565-006-0039-7

27. Richards JM, Boyter AC, Nathwani D. A jaundiced farmer. Scott Med J 1996;41:176-7. doi $10.1177 / 003693309604100607$
28. Wu YQ, Wang JD, Chen JS, Chung SC, Hwang SY. Occupational risk of decreased plasma cholinesterase among pesticide production workers in Taiwan. Am J Ind Med 1989;16:659-66. doi: 10.1002/ajim.4700160605

29. Kundiev Yu I, Krasnyuk EP, Viter VPH. Specific features of the changes in the health status of female workers exposed to pesticides in greenhouses. Toxicol Lett 1986;33:85-9. doi: 10.1016/0378-4274(86)90073-1

30. Malekirad AA, Faghih M, Mirabdollahi M, Kiani M, Fathi A, Abdollahi M. Neurocognitive, mental health, and glucose disorders in farmers exposed to organophosphorus pesticides. Arh Hig Rada Toksikol 2013;64:1-8. doi: 10.2478/100041254-64-2013-2296

31. Avsarogullari L, Ikizceli I, Sungur M, Sözüer E, Akdur O, Yücei M. Acute amitraz poisoning in adults: clinical features, laboratory findings, and management. Clin Toxicol (Phila) 2006;44:19-23. PMID: 16496489

32. Mikhail TH, Aggour N, Awadallah R, Boulos MN, ElDessoukey EA, KarimaAI. Acute toxicity of organophosphorus and organochlorine insecticides in laboratory animals. Z Ernährungswiss 1979;18:258-68. PMID: 95070

33. Goel A, Dani V, Dhawan DK. Protective effects of zinc on lipid peroxidation, antioxidant enzymes and hepatic histoarchitecture in chlorpyrifos-induced toxicity. Chem Biol Interact 2005;156:131-40. doi: 10.1016/j.cbi.2005.08.

34. Ezzi L, Belhadj Salah I, Haouas Z, Sakly A, Grissa I, Chakroun S, Kerkeni E, Hassine M, Mehdi M, Ben Cheikh H. Histopathological and genotoxic effects of chlorpyrifos in rats. Environ Sci Pollut Res Int 2016;23:4859-67. doi: 10.1007/s11356-015-5722-x

35. Topal A, Atamanalp M, Oruc E, Demir Y, Beydemir S, Isik A. In vivo changes in carbonic anhydrase activity and histopathology of gill and liver tissues after acute exposure to chlorpyrifos in rainbow trout. Arh Hig Rada Toksikol. 2014;65:377-85. doi: 10.2478/10004-1254-65-2014-2547

36. Sharma D, Sangha GK. Triazophos induced oxidative stress and histomorphological changes in liver and kidney of female albino rats. Pestic Biochem Physiol 2014;110:71-80. doi: 10.1016/j.pestbp.2014.03.003

37. Banaee M, Sureda A, Mirvaghefi AR, Ahmadi K. Biochemical and histological changes in the liver tissue of rainbow trout (Oncorhynchus mykiss) exposed to sub-lethal concentrations of diazinon. Fish Physiol Biochem 2013;39:489-501. doi: 10.1007/s10695-012-9714-1

38. Zhuang XM, Wei X, Tan Y, Xiao WB, Yang HY, Xie JW, Lu $\mathrm{C}, \mathrm{Li} \mathrm{H}$. Contribution of carboxylesterase and cytochrome $\mathrm{P} 450$ to the bioactivation and detoxification of isocarbophos and its enantiomers in human liver microsomes. Toxicol Sci 2014;140:40-8. doi: 10.1093/toxsci/kfu067

39. Beydilli H, Yilmaz N, Cetin ES, Topal Y, Celik OI, Sahin C, Topal H, Cigerci IH, Sozen H. Evaluation of the protective effect of silibinin against diazinon induced hepatotoxicity and free-radical damage in rat liver. Iran Red Crescent Med J 2015;17(4):e25310. doi: 10.5812/ircmj.17(4)2015.25310

40. Saraei F, Sadoughi M, Kaka G, Sadraie SH, Foaddodini M. Study of the effects of diazinon on fetal liver in BALB/c mice. Iran Red Crescent Med J 2016;18(4):e28076. doi: 10.5812/ircmj.28076

41. Baconi DL, Barca M, Manda G, Ciobanu AM, Balalau C. Investigation of the toxicity of some organophosphorus pesticides in a repeated dose study in rats. Rom J Morphol Embryol 2013;54:349-56. PMID: 23771080 
42. Naraharisetti SB, Aggarwal M, Ranganathan V, Sarkar SN, Kataria M, Malik JK. Effects of simultaneous repeated exposure at high levels of arsenic and malathion on hepatic drug-biotransforming enzymes in broiler chickens. Environ Toxicol Pharmacol 2009;28:213-8. doi: 10.1016/j. etap.2009.04.006

43. Chakraborty D, Bhattacharyya A, Majumdar K, Chatterjee K, Chatterjee S, Sen A, Chatterjee GC. Studies on L-ascorbic acid metabolism in rats under chronic toxicity due to organophosphorus insecticides: effects of supplementation of L-ascorbic acid in high doses. J Nutr 1978;108:973-80. PMID: 650299

44. Rezg R, Mornagui B, El-Arbi M, Kamoun A, El-Fazaa S, Gharbi N. Effect of subchronic exposure to malathion on glycogen phosphorylase and hexokinase activities in rat liver using native PAGE. Toxicology 2006;223:9-14. doi: 10.1016/j.tox.2006.02.020

45. Ahmadipour A, Sharififar F, Pournamdari M, Bamkan AM, Hosseini A, Afrapoli FM, Karami-Mohajer S. Hepatoprotective effect of Zataria Multiflora Boiss against malathion-induced oxidative stress in male rats. Orient Pharm Exp Med 2016;16:287-93. doi: 10.1007/s13596-016-0238-6

46. Undeger U, Institoris L, Siroki O, Nehéz M, Dési I. Simultaneous geno- and immunotoxicological investigations for early detection of organophosphate toxicity in rats. Ecotoxicol Environ Saf 2000;45:43-8. doi: 10.1006/ eesa.1999.1844

47. Fanta E, Rios FS, Romao S, Vianna AC, Freiberger S. Histopathology of the fish Corydoras paleatus contaminated with sublethal levels of organophosphorus in water and food. Ecotoxicol Environ Saf 2003;54:119-30. doi: 10.1016/S01476513(02)00044-1

48. Li B, Ma Y, Zhang YH. Oxidative stress and hepatotoxicity in the frog, Rana chensinensis, when exposed to low doses of trichlorfon. J Environ Sci Health B 2017;52:476-82. doi: 10.1080/03601234.2017.1303321

49. Lu L, Wang X, Lang L, Fu F. Protective effect of reduced glutathione on the liver injury induced by acute omethoate poisoning. Environ Toxicol Pharmacol 2010;30:279-83. doi: 10.1016/j.etap.2010.08.001

50. Hernández AF, Amparo Gómez M, Pérez V, García-Lario JV, Pena G, Gil F, López O, Rodrigo L, Pino G, Pla A. Influence of exposure to pesticides on serum components and enzyme activities of cytotoxicity among intensive agriculture farmers. Environ Res 2006;102:70-6. doi: 10.1016/j. envres.2006.03.002

51. Hariri AT, Moallem SA, Mahmoudi M, Memar B, Hosseinzadeh $\mathrm{H}$. Sub-acute effects of diazinon on biochemical indices and specific biomarkers in rats: protective effects of crocin and safranal. Food Chem Toxicol 2010;48:2803-8. doi: 10.1016/j.fct.2010.07.010

52. Gomes J, Dawodu AH, Lloyd O, Revitt DM, Anilal SV. Hepatic injury and disturbed amino acid metabolism in mice following prolonged exposure to organophosphorus pesticides. Hum Exp Toxicol 1999;18:33-7. doi: 10.1177/096032719901800105

53. Goel A, Chauhan D, Dhawan D. Protective effects of zinc in chlorpyrifos induced hepatotoxicity. Biol Trace Elem Res 2000;74:171-83. doi: 10.1385/BTER:74:2:171

54. Elhalwagy ME, Darwish NS, Zaher EM. Prophylactic effect of green tea polyphenols against liver and kidney injury induced by fenitrothion insecticide. Pest Biochem Physiol 2008;91:81-9. doi: 10.1016/j.pestbp.2008.01.006

55. Mostafalou S, Abdollahi M. Pesticides: an update of human exposure and toxicity. Arch Toxicol 2017; 91(2):549-99. doi: 10.1007/s00204-016-1849-x.

56. Benedetti AsL, Vituri CdL, Trentin AG, Domingues MAC, Alvarez-Silva M. The effects of sub-chronic exposure of Wistar rats to the herbicide Glyphosate-Biocarb ${ }^{\circledR}$. Toxicol lett 2004;153:227-32. doi: 10.1016/j.toxlet.2004.04.008

57. Begum G. Carbofuran insecticide induced biochemical alterations in liver and muscle tissues of the fish Clarias batrachus (linn) and recovery response. Aquat Toxicol 2004;66:83-92. doi: 10.1016/j.aquatox.2003.08.002

58. Reena K, Ajay K, Sharma CB. Haematological changes induced by dimethoate in rat. Arh Hig Rada Toksikol 1989;40:23-7. PMID: 2803006

59. Kaur S, Dhanju CK. Enzymatic changes induced by some organophosphorus pesticides in female rats. Indian J Exp Biol 2004;42:1017-9. PMID: 15511008

60. Venkateswara Rao J. Sublethal effects of an organophosphorus insecticide (RPR-II) on biochemical parameters of tilapia, Oreochromis mossambicus. Comp Biochem Physiol C Toxicol Pharmacol 2006;143:492-8. doi: 10.1016/j. cbpc.2006.05.001

61. Altuntas I, Delibas N, Demirci M, Kilinc I, Tamer N. The effects of methidathion on lipid peroxidation and some liver enzymes: role of vitamins E and C. Arch Toxicol 2002;76:4703. doi: 10.1007/s00204-002-0359-1

62. Enan EE, El-Sebae AH, Enan OH, El-Fiki S. In-vivo interaction of some organophosphorus insecticides with different biochemical targets in white rats. J Environ Sci Health B 1982;17:549-70. doi: 10.1080/03601238209372341

63. El-Demerdash FM, Nasr HM. Antioxidant effect of selenium on lipid peroxidation, hyperlipidemia and biochemical parameters in rats exposed to diazinon. J Trace Elem Med Biol 2014;28:89-93. doi: 10.1016/j.jtemb.2013.10.001

64. Jayusman PA, Budin SB, Ghazali AR, Taib IS, Louis SR. Effects of palm oil tocotrienol-rich fraction on biochemical and morphological alterations of liver in fenitrothion-treated rats. Pak J Pharm Sci 2014;27:1873-80. PMID: 25362611

65. Hou YX, Liu SW, Wang LW, Wu SH. Physiopathology of multiple organ dysfunction in severely monocrotophospoisoned rabbits. Chem Biol Interact 2017;278:9-14. doi: 10.1016/j.cbi.2017.08.016

66. Patil JA, Patil AJ, Govindwar SP. Biochemical effects of various pesticides on sprayers of grape gardens. Indian J Clinic Biochem 2003;18:16-22. doi: 10.1007/BF02867362

67. Azmi MA, Naqvi S, Azmi MA, Aslam M. Effect of pesticide residues on health and different enzyme levels in the blood of farm workers from Gadap (rural area) Karachi-Pakistan. Chemosphere 2006;64:1739-44. doi: 10.1016/j. chemosphere.2006.01.016

68. Jyothi B, Narayan G. Certain pesticide-induced carbohydrate metabolic disorders in the serum of freshwater fish Clarias batrachus (Linn.). Food Chem Toxicol 1999;37:417-21. doi: 10.1016/S0278-6915(99)00020-4

69. Araoud M, Neffeti F, Douki W, Khaled L, Najjar MF, Kenani A, Houas Z. Toxic effects of methamidophos on paraoxonase 1 activity and on rat kidney and liver and ameliorating effects of alpha-tocopherol. Environ Toxicol 2016;31:842-54. doi: 10.1002/tox.22095 
70. Li T, Chiang JY. Bile acid signaling in metabolic disease and drug therapy. Pharmacol Rev 2014;66:948-83. doi: 10.1124/ pr.113.008201

71. Goel A, Dhawan DK. Zinc supplementation prevents liver injury in chlorpyrifos-treated rats. Biol Trace Elem Res 2001;82:185-200. doi: 10.1385/BTER:82:1-3:185

72. Farghaly M, Mahdy F, Taha H, Fathy U. Behavior of the organophosphorus insecticide fenitrothion in stored faba beans and its biological effects towards experimental animals. J Environ Sci Health B 2007;42:655-62. doi: $10.1080 / 03601230701465718$

73. Cecchi A, Rovedatti MG, Sabino G, Magnarelli GG. Environmental exposure to organophosphate pesticides: assessment of endocrine disruption and hepatotoxicity in pregnant women. Ecotoxicol Environ Saf 2012;80:280-7. doi: 10.1016/j.ecoenv.2012.03.008

74. Koundinya PR, Ramamurthi R. Effect of organophosphate pesticide Sumithion (Fenitrothion) on some aspects of carbohydrate metabolism in a freshwater fish, Sarotherodon (Tilapia) mossambicus (Peters). Experientia 1979;35:1632-3. PMID: 520481

75. Lasram MM, El-Golli N, Lamine AJ, Douib IB, Bouzid K, Annabi A, El Fazaa S, Abdelmoula J, Gharbi N. Changes in glucose metabolism and reversion of genes expression in the liver of insulin-resistant rats exposed to malathion. The protective effects of N-acetylcysteine. Gen Comp Endocrinol 2015;215:88-97. doi: 10.1016/j.ygcen.2014.10.002

76. Srivastava AK, Mishra J. Effects of fenthion on the blood and tissue chemistry of a teleost fish (Heteropneustes fossilis). J Comp Pathol 1983;93:27-31. doi: 10.1016/00219975(83)90040-3

77. Abdollahi M, Donyavi M, Pournourmohammadi S, Saadat M. Hyperglycemia associated with increased hepatic glycogen phosphorylase and phosphoenolpyruvate carboxykinase in rats following subchronic exposure to malathion. Comp Biochem Physiol C Toxicol Pharmacol 2004;137:343-7. doi: 10.1016/j.cca.2004.03.009

78. Teimouri F, Amirkabirian N, Esmaily H, Mohammadirad A, Aliahmadi A, Abdollahi M. Alteration of hepatic cells glucose metabolism as a non-cholinergic detoxication mechanism in counteracting diazinon-induced oxidative stress. Hum Exp Toxicol 2006;25:697-703. doi: 10.1177/0960327106075064

79. Basiri S, Esmaily H, Vosough-Ghanbari S, Mohammadirad A, Yasa N, Abdollahi M. Improvement by Satureja khuzestanica essential oil of malathion-induced red blood cells acetylcholinesterase inhibition and altered hepatic mitochondrial glycogen phosphorylase and phosphoenolpyruvate carboxykinase activities. Pestic Biochem Physiol 2007;89:124-9. doi: 10.1016/j. pestbp.2007.04.006

80. Joshi AK, Rajini PS. Reversible hyperglycemia in rats following acute exposure to acephate, an organophosphorus insecticide: role of gluconeogenesis. Toxicology 2009;257:405. doi: 10.1016/j.tox.2008.12.006

81. Lari P, Rashedinia M, Abnous K, Hosseinzadeh H. Alteration of protein profile in rat liver of animals exposed to subacute diazinon: a proteomic approach. Electrophoresis 2014;35:1419-27. doi: 10.1002/elps.20130047

82. Romero-Navarro G, Lopez-Aceves T, Rojas-Ochoa A, Fernandez Mejia C. Effect of dichlorvos on hepatic and pancreatic glucokinase activity and gene expression, and on insulin mRNA levels. Life Sci 2006;78:1015-20. doi: 10.1016/j.lfs.2005.06.010

83. Husain K, Mirza MA, Matin MA. Convulsions as the etiology of lactic acidosis in acute diazinon toxicity in rats. Toxicol Lett. 1987; 37: 257-61. doi: 10.1016/0378-4274(87)90140-8

84. Patel PB, Chakrabarti $\mathrm{CH}$. Changes in the activity of some hepatic enzymes during organophosphorus insecticideacephate (orthene) treatment in albino rats. Indian J Physiol Pharmacol 1982;26:311-6. PMID: 7152641

85. Das BK, Mukherjee SC. Chronic toxic effects of quinalphos on some biochemical parameters in Labeo rohita (Ham.). Toxicol Lett 2000;114:11-8. doi: 10.1016/S03784274(99)00185-X

86. Sancho E, Ferrando MD, Andreu E. Sublethal effects of an organophosphate insecticide on the European eel, Anguilla anguilla. Ecotoxicol Environ Saf 1997;36:57-65. doi: 10.1006/eesa.1996.1488

87. Mukhamedzhanov EK, Apsatarova RA, Ionina MP. [Effect of organophosphate pesticides on oxidative metabolism against a background of a protein-balanced and -imbalanced diet, in Russian]. Vopr Pitan 1981;(1):56-60. PMID: 6784341

88. Kaur P, Radotra B, Minz RW, Gill K. Impaired mitochondrial energy metabolism and neuronal apoptotic cell death after chronic dichlorvos (OP) exposure in rat brain. Neurotoxicology 2007;28:1208-19. doi: 10.1016/j.neuro.2007.08.001

89. Karami-Mohajeri S, Abdollahi M. Mitochondrial dysfunction and organophosphorus compounds. Toxicol Appl Pharmacol 2013;270:39-44. doi: 10.1016/j.taap.2013.04.001

90. Spetale MR, Morisoli LS, Rodriguez Garay EA. The effect of organophosphorus compounds on respiration by rat liver mitochondria. Farmaco Sci 1977;32:116-22. PMID: 858381

91. Yamano T, Morita S. Effects of pesticides on isolated rat hepatocytes, mitochondria, and microsomes. Arch Environ Contam Toxicol. 1993;25(2):271-8.

92. Shabarchin EI, Kruglyakova KE, Gendel LY, Kabanov VV. Influence of metaphos on the structuro-functional organization of mitochondrial membranes. Biol Bull Acad Sci USSR 1979;6:788-93. PMID: 233298

93. Binukumar BK, Bal A, Kandimalla R, Sunkaria A, Gill KD. Mitochondrial energy metabolism impairment and liver dysfunction following chronic exposure to dichlorvos. Toxicology 2010;270:77-84. doi: 10.1016/j.tox.2010.01.017

94. Mohammad Abdollahi, Maryam Baeeri, Karami-Mohajeri S. Effects of acute malathion exposure on liver mitochondrial respiratory chain enzymes in rats. Toxicol Int 2015;22:52-7. doi: 10.22506/ti/2015/v22/i2/137656

95. Shafiee H, Mohammadi H, Rezayat SM, Hosseini A, Baeeri M, Hassani S, Mohammadirad A, Bayrami Z, Abdollahi M. Prevention of malathion-induced depletion of cardiac cells mitochondrial energy and free radical damage by a magnetic magnesium-carrying nanoparticle. Toxicol Mech Methods 2010;20:538-43. doi: 10.3109/15376516.2010.518173

96. Massicotte C, Knight K, Van der Schyf CJ, Jortner BS, Ehrich M. Effects of organophosphorus compounds on ATP production and mitochondrial integrity in cultured cells. Neurotox Res 2005;7:203-17. PMID: 15897155

97. Venkatesh S, Ramachandran A, Zachariah A, Oommen A. Mitochondrial ATP synthase inhibition and nitric oxide are involved in muscle weakness that occurs in acute exposure of rats to monocrotophos. Toxicol Mech Methods 2009;19:239-45. doi: 10.1080/15376510802455354 
98. Kashyap MP, Singh AK, Siddiqui MA, Kumar V, Tripathi VK, Khanna VK, Yadav S, Jain SK, Pant AB. Caspase cascade regulated mitochondria mediated apoptosis in monocrotophos exposed PC12 cells. Chem Res Toxicol 2010;23:1663-72. doi: 10.1021/tx100234m

99. Karami-Mohajeri S, Hadian M, Fouladdel S, Azizi E, Ghahramani M, Hosseini R, Abdollahi M. Mechanisms of muscular electrophysiological and mitochondrial dysfunction following exposure to malathion, an organophosphorus pesticide. Hum Exp Toxicol 2014;33:251-63. doi: 10.1177/0960327113493300

100. Middlemore-Risher ML, Adam BL, Lambert NA, Terry AV, Jr. Effects of chlorpyrifos and chlorpyrifos-oxon on the dynamics and movement of mitochondria in rat cortical neurons. J Pharmacol Exp Ther 2011;339:341-9. doi: 10.1124/jpet.111.184762

101. Sastry KV, Siddiqui AA. Chronic toxic effects of the carbamate pesticide sevin on carbohydrate metabolism in a freshwater snakehead fish, Channa punctatus. Toxicol Lett 1982;14:123-30. doi: 10.1016/0378-4274(82)90019-4

102. Lynch CJ, Fox H, Hazen SA, Stanley BA, Dodgson S, Lanoue KF. Role of hepatic carbonic anhydrase in de novo lipogenesis. Biochem J 1995;310:197-202. PMCID PMC1135873

103. Huang Q, Huang HQ. Alterations of protein profile in zebrafish liver cells exposed to methyl parathion: a membrane proteomics approach. Chemosphere 2012;87:68-76. doi: 10.1016/j.chemosphere.2011.11.061

104. Nagaraju R, Joshi AK, Rajini PS. Organophosphorus insecticide, monocrotophos, possesses the propensity to induce insulin resistance in rats on chronic exposure. J Diabetes 2015;7:47-59. doi: 10.1111/1753-0407.12158

105. Xu W, Liu W, Shao X, Jiang G, Li X. Effect of trichlorfon on hepatic lipid accumulation in crucian carp Carassius auratus gibelio. J Aquat Anim Health 2012;24:185-94. doi: 10.1080/08997659.2012.675937

106. Takeuchi S, Matsuda T, Kobayashi S, Takahashi T, Kojima H. In vitro screening of 200 pesticides for agonistic activity via mouse peroxisome proliferator-activated receptor (PPAR) alpha and PPARgamma and quantitative analysis of in vivo induction pathway. Toxicol Appl Pharmacol 2006;217:23544. doi: 10.1016/j.taap.2006.08.011

107. Bandyopadhyay U, Das D, Banerjee RK. Reactive oxygen species: oxidative damage and pathogenesis. Curr Sci 1999;77:658-66.

108. Sahin I, Onbasi K, Sahin H, Karakaya C, Ustun Y, Noyan T. The prevalence of pancreatitis in organophosphate poisonings. Hum Exp Toxicol 2002;21:175-7. DOI: $10.1191 / 0960327102 \mathrm{ht} 234 \mathrm{cr}$

109. Muniz JF, McCauley L, Scherer J, Lasarev M, Koshy M, Kow YW, Nazar-Stewart V, Kisby GE. Biomarkers of oxidative stress and DNA damage in agricultural workers: a pilot study. Toxicol Appl Pharmacol 2008;227:97-107. doi: 10.1016/j.taap.2007.10.027

110. Bagchi D, Bagchi M, Hassoun E, Stohs S. In vitro and in vivo generation of reactive oxygen species, DNA damage and lactate dehydrogenase leakage by selected pesticides. Toxicology $1995 ; 104: 129-40$. do i : 10.1016/0300-483X(95)03156-A

111. Yamano T, Morita S. Hepatotoxicity of trichlorfon and dichlorvos in isolated rat hepatocytes. Toxicology 1992;76:6977. doi: 10.1016/0300-483X(92)90019-B
112. Lopez O, Hernandez AF, Rodrigo L, Gil F, Pena G, Serrano JL, Parrón T, Villanueva E, Pla A. Changes in antioxidant enzymes in humans with long-term exposure to pesticides. Toxicol Lett 2007;171:146-53. doi: 10.1016/j. toxlet.2007.05.004

113. Bagchi D, Balmoori J, Bagchi M, Ye X, Williams CB, Stohs SJ. Comparative effects of TCDD, endrin, naphthalene and chromium (VI) on oxidative stress and tissue damage in the liver and brain tissues of mice. Toxicology 2002;175:73-82. doi: 10.1016/S0300-483X(02)00062-8

114. Vidyasagar J, Karunakar N, Reddy M, Rajnarayana K, Surender T, Krishna D. Oxidative stress and antioxidant status in acute organophosphorous insecticide poisoning. Indian J Pharmacol 2004;36:76-9.

115. Banerjee B, Seth V, Bhattacharya A, Pasha S, Chakraborty A. Biochemical effects of some pesticides on lipid peroxidation and free-radical scavengers. Toxicol Lett 1999;107:33-47. doi: 10.1016/S0378-4274(99)00029-6

116. Akhgari M, Abdollahi M, Kebryaeezadeh A, Hosseini R, Sabzevari O. Biochemical evidence for free radical-induced lipid peroxidation as a mechanism for subchronic toxicity of malathion in blood and liver of rats. Hum Exp Toxicol 2003;22:205-11. doi: 10.1191/0960327103ht346oa

117. Kovacic P. Mechanism of organophosphates (nerve gases and pesticides) and antidotes: electron transfer and oxidative stress. Curr Med Chem 2003;10:2705-9. PMID: 14529460

118. Sharma Y, Bashir S, Irshad M, Nag TC, Dogra TD. Dimethoate-induced effects on antioxidant status of liver and brain of rats following subchronic exposure. Toxicology 2005;215:173-81. doi: 10.1016/j.tox.2005.06.029

119. Nili-Ahmadabadi A, Tavakoli F, Hasanzadeh G, Rahimi H, Sabzevari O. Protective effect of pretreatment with thymoquinone against Aflatoxin $\mathrm{B}(1)$ induced liver toxicity in mice. Daru 2011;19:282-7. PMCID: PMC3304388

120. Rahimi HR, Agin K, Shadnia S, Hassanian-Moghaddam H, Oghazian MB. Clinical and biochemical analysis of acute paint thinner intoxication in adults: a retrospective descriptive study. Toxicol Mech Methods 2015;25:42-7. doi: 10.3109/15376516.2014.97538

121. Ma J, Liu Y, Niu D, Li X. Effects of chlorpyrifos on the transcription ofCYP3AcDNA, activity of acetylcholinesterase, and oxidative stress response of goldfish (Carassius auratus). Environ Toxicol 2015;30:422-9. doi: 10.1002/tox.21918

122. Verma RS, Srivastava N. Effect of chlorpyrifos on thiobarbituric acid reactive substances, scavenging enzymes and glutathione in rat tissues. Indian J Biochem Biophys 2003;40:423-8. PMID: 22900370

123. Messarah M, Amamra W, Boumendjel A, Barkat L, Bouasla I, Abdennour C, Boulakoud MS, Feki AE. Ameliorating effects of curcumin and vitamin $\mathrm{E}$ on diazinon-induced oxidative damage in rat liver and erythrocytes. Toxicol Ind Health 2013;29(1):77-8. doi: 10.1177/0748233712446726

124. Uner N, Sevgiler Y, Durmaz H, Piner P. In vivo alterations in glutathione-related processes, lipid peroxidation, and cholinesterase enzyme activities in the liver of diazinonexposed oreochromis niloticus. Toxicol Mech Methods 2007;17(6):317-24. doi: 10.1080/15376510601031927

125. Oruc E. Effects of diazinon on antioxidant defense system and lipid peroxidation in the liver of Cyprinus carpio (L.). Environ Toxicol. 2011;26(6):571-8. doi: 10.1002/tox.20573

126. Xu MY, Wang P, Sun YJ, Wang HP, Liang YJ, Zhu L, Wu, YJ. Redox status in liver of rats following subchronic 
exposure to the combination of low dose dichlorvos and deltamethrin. Pestic Biochem Physiol 2015;124:60-5. doi: 10.1016/j.pestbp.2015.04.005

127. Ayed-Boussema I, Rjiba K, Moussa A, Mnasri N, Bacha H. Genotoxicity associated with oxidative damage in the liver and kidney of mice exposed to dimethoate subchronic intoxication. Environ Sci Pollut Res Int 2012;19:458-66. doi: 10.1007/s11356-011-0588-z

128. Abd El-Gawad EA, Abdel Hamid OM. Effect of vitamin C dietary supplementation in reducing the alterations induced by fenitrothion in Oreochromis niloticus Fish Physiol Biochem 2014;40(3):787-96. doi: 10.1007/s10695-0139885-4

129. Lasram MM, Lamine AJ, Dhouib IB, Bouzid K, Annabi A, Belhadjhmida N, Ahmed MB, El Fazaa S, Abdelmoula J, Gharbi N. Antioxidant and anti-inflammatory effects of $\mathrm{N}$-acetylcysteine against malathion-induced liver damages and immunotoxicity in rats. Life Sci 2014;107(1-2):50-8. doi: 10.1016/j.lfs.2014.04.033

130. Edwards FL, Yedjou CG, Tchounwou PB. Involvement of oxidative stress in methyl parathion and parathion-induced toxicity and genotoxicity to human liver carcinoma $\left(\mathrm{HepG}_{2}\right)$ cells. Environ Toxicol 2013;28:342-8. doi: 10.1002/ tox. 20725

131. Moore PD, Yedjou CG, Tchounwou PB. Malathion-induced oxidative stress, cytotoxicity, and genotoxicity in human liver carcinoma $\left(\mathrm{HepG}_{2}\right)$ cells. Environ Toxicol 2010;25:221-6. doi: $10.1002 /$ tox. 20492

132. Tuzmen N, Candan N, Kaya E, Demiryas N. Biochemical effects of chlorpyrifos and deltamethrin on altered antioxidative defense mechanisms and lipid peroxidation in rat liver. Cell Biochem Funct 2008;26:119-24. doi: 10.1002/ cbf. 1411

133. Uner, N., Y. Sevgiler, Piner P, Durmaz H. Modulation of fenthion-induced oxidative effects by BSO in the liver of Cyprinus carpio L. Drug Chem Toxicol 2008;31(3):353-369. doi: $10.1080 / 01480540802171233$

134. Toledo-Ibarra GA, Diaz Resendiz KJ, Ventura-Ramon GH, Gonzalez-Jaime F, Vega-Lopez A, Becerril-Villanueva E, Pavon L, Giron-Perez MI. Oxidative damage in gills and liver in Nile tilapia (Oreochromis niloticus) exposed to diazinon. Comp Biochem Physiol A Mol Integr Physiol 2016;200:3-8. doi: 10.1016/j.cbpa.2016.05.007

135. Woo S, Yum S, Kim DW, Park HS. Transcripts level responses in a marine medaka (Oryzias javanicus) exposed to organophosphorus pesticide. Comp Biochem Physiol C Toxicol Pharmacol 2009;149:427-32. doi: 10.1016/j. cbpc.2008.10.100

136. Smith RA, Porteous CM, Coulter CV, Murphy MP. Selective targeting of an antioxidant to mitochondria. Eur J Biochem 1999;263:709-16. doi: 10.1046/j.1432-1327.1999.00543.x

137. Marchi S, Giorgi C, Suski JM, Agnoletto C, Bononi A, Bonora M, De Marchi E, Missiroli S, Patergnani S, Poletti F, Rimessi A, Duszynski J, Wieckowski MR, Pinton P. Mitochondria-ros crosstalk in the control of cell death and aging. J Signal Transduct 2012;2012: 329635. doi: $10.1155 / 2012 / 329635$

138. Baghaei A, Hajimohammadi N, Baeeri M, Mohammadirad A, Hassani S, Abdollahi M. On the protection of ALP cardiovascular toxicity by a novel mixed herbal medicine; role of oxidative stress and cellular ATP. Asian J Anim Vet Adv 2014;9:302-11. doi: 10.3923/ajava.2014.302.311
139. Kowaltowski AJ, Vercesi AE. Mitochondrial damage induced by conditions of oxidative stress. Free Radic Biol Med 1999;26:463-71. doi: 10.1016/S0891-5849(98)00216-0

140. Jo SH, Son MK, Koh HJ, Lee SM, Song IH, Kim YO, Lee YS, Jeong KS, Kim WB, Park JW, Song BJ, Huh TL. Control of mitochondrial redox balance and cellular defense against oxidative damage by mitochondrial $\mathrm{NADP}^{+}$-dependent isocitrate dehydrogenase. J Biol Chem 2001;276:16168-76. doi: 10.1074/jbc.M010120200

141. Fiskum G, Starkov A, Polster BM, Chinopoulos C. Mitochondrial mechanisms of neural cell death and neuroprotective interventions in Parkinson's disease. Ann N Y Acad Sci 2003;991:111-9. doi: 10.1111/j.1749-6632.2003. tb07469.x

142. Yazdinezhad A, Abbasian M, Hojjat Hosseini S, Naserzadeh P, Agh-Atabay AH, Hosseini MJ. Protective effects of Ziziphora tenuior extract against chlorpyrifos induced liver and lung toxicity in rat: Mechanistic approaches in subchronic study. Environ Toxicol 2017;32:2191-2202. doi: 10.1002/ tox. 22432

143. Lari P, Rashedinia M, Abnous K, Hosseinzadeh H. Crocin improves lipid dysregulation in subacute diazinon exposure through ERK1/2 pathway in rat liver. Drug Res (Stuttg) 2013;64:301-5. doi: 10.1055/s-0033-1357196

144. Butler AM, Murray M. Inhibition and inactivation of constitutive cytochromes $\mathrm{P} 450$ in rat liver by parathion. Mol Pharmacol 1993;43:902-8. PMID: 8316222

145. Zhuang Z, Tang M, Zheng Y, Hu L, Lin F. Effect of dimethoate on the activity of hepatic CYP450 based on pharmacokinetics of probe drugs. Pharmacology 2015;95:24350. doi: 10.1159/00038192

146. Weber RP, Coon JM, Triolo AJ. Effect of the organophosphate insecticide parathion and its active metabolite paraoxon on the metabolism of benzo(a)pyrene in the rat. Cancer Res 1974;34:947-52. PMID: 4823904

147. Van der Meer MJ, Hundt HK, Muller FO. Inhibition of atropine metabolism by organophosphate pesticides. Hum Toxicol 1983;2:637-40. PMID: 6642521

148. Takeuchi S, Iida M, Yabushita H, Matsuda T, Kojima H. In vitro screening for aryl hydrocarbon receptor agonistic activity in 200 pesticides using a highly sensitive reporter cell line, DR-EcoScreen cells, and in vivo mouse liver cytochrome P450-1A induction by propanil, diuron and linuron. Chemosphere 2008;74:155-65. doi: 10.1016/j. chemosphere.2008.08.015

149. Long M, Laier P, Vinggaard AM, Andersen HR, Lynggaard J, Bonefeld-Jorgensen EC. Effects of currently used pesticides in the AhR-CALUX assay: comparison between the human TV101L and the rat H4IIE cell line. Toxicology 2003;194:77-93. doi: 10.1016/j.tox.2003.08.001

150. Hankinson O. The aryl hydrocarbon receptor complex. Annu Rev Pharmacol Toxicol 1995;35:307-40. doi: 10.1146/ annurev.pa.35.040195.001515

151. Salvadori D, Ribeiro L, Pereira C, Becak W. Cytogenetic effects of malathion insecticide on somatic and germ cells of mice. Mutat Res 1988;204:283-7. doi: 10.1016/01651218(88)90101-2

152. Bolognesi C. Genotoxicity of pesticides: a review of human biomonitoring studies. Mutat Res 2003;543:251-72. doi: $10.1016 / \mathrm{S} 1383-5742(03) 00015-2$ 
153. Flessel P, Quintana P, Hooper K. Genetic toxicity of malathion: a review. Environ Mol Mutagen 1993;22:7-17. doi: 10.1002/em.2850220104

154. Muranli FD, Kanev M, Ozdemir K. Genotoxic effects of diazinon on human peripheral blood lymphocytes. Arh Hig Rada Toksikol 2015;66:153-8. doi: 10.1515/aiht-2015-662584

155. Gilot-Delhalle J, Colizzi A, Moutschen J, MoutschenDahmen M. Mutagenicity of some organophosphorus compounds at the ade6 locus of Schizosaccharomyces pombe. Mutat Res 1983;117:139-48. PMID: 6835257

156. Shadnia S, Azizi E, Hosseini R, Khoei S, Fouladdel S, Pajoumand A, Jalali N, Abdollahi M. Evaluation of oxidative stress and genotoxicity in organophosphorus insecticide formulators. Hum Exp Toxicol 2005;24:439-45. doi: 10.1191/0960327105ht549oa

157. Grover P, Danadevi K, Mahboob M, Rozati R, Banu BS, Rahman M. Evaluation of genetic damage in workers employed in pesticide production utilizing the Comet assay. Mutagenesis 2003;18:201-5. doi: 10.1093/mutage/18.2.201

158. Mehta A, Verma RS, Srivastava N. Chlorpyrifos-induced DNA damage in rat liver and brain. Environ Mol Mutagen 2008;49:426-33. doi: 10.1002/em.20397

159. Saquib Q, Attia SM, Siddiqui MA, Aboul-Soud MA, AlKhedhairy AA, Giesy JP, Musarrat J. Phorate-induced oxidative stress, DNA damage and transcriptional activation of p53 and caspase genes in male Wistar rats. Toxicol Appl Pharmacol 2012;259:54-65. doi: 10.1016/j.taap.2011.12.006

160. Hreljac I, Zajc I, Lah T, Filipic M. Effects of model organophosphorous pesticides on DNA damage and proliferation of $\mathrm{HepG}_{2}$ cells. Environ Mol Mutagen 2008;49:360-7. doi: 10.1002/em.20392

161. Reuber MD. Carcinogenicity of dimethoate. Environ Res 1984;34:193-211. doi: 10.1016/0013-9351(84)90089-6

162. Salmon AG. IARC monographs on the Evaluation of Carcinogenic Risk of Chemicals to Humans. Br J Ind Med 1985;42(3):214. PMCID: PMC1007456

163. Bonner MR, Coble J, Blair A, Freeman LEB, Hoppin JA, Sandler DP, Alavanja MC. Malathion exposure and the incidence of cancer in the agricultural health study. Am J Epidemiol 2007;166:1023-34. doi: 10.1093/aje/kwm182
164. Wei M, Wanibuchi H, Yamamoto S, Li W, Fukushima S. Urinary bladder carcinogenicity of dimethylarsinic acid in male F344 rats. Carcinogenesis 1999;20:1873-6. PMID: 10469637

165. Rahimi HR, Arastoo M, Ostad SN. A Comprehensive review of Punica granatum (Pomegranate) properties in toxicological, pharmacological, cellular and molecular biology researches. Iran J Pharm Res 2012;11:385-400. PMCID: PMC3832175

166. Balouchzadeh A, Rahimi H, Ebadollahi-Natanzi A, MinaeiZangi B, Sabzevari O. Aqueous extract of Iranian green tea prevents lipid peroxidation and chronic ethanol liver toxicity in rat. J Pharm Toxicol 2011;6:691-700. doi: 10.3923/ jpt.2011.691.700

167. Shokrzadeh M, Ahangar N, Abdollahi M, Shadboorestan A, Omidi M, Payam SS. Potential chemoprotective effects of selenium on diazinon-induced DNA damage in rat peripheral blood lymphocyte. Hum Exp Toxicol 2013;32:759-65. doi: $10.1177 / 0960327112468179$

168. Tehrani H, Halvaie Z, Shadnia S, Soltaninejad K, Abdollahi M. Protective effects of $\mathrm{N}$-acetylcysteine on aluminum phosphide-induced oxidative stress in acute human poisoning. Clin Toxicol (Phila) 2013;51:23-8. doi: 10.3109/15563650.2012.743029

169. Ranjbar A, Ghahremani MH, Sharifzadeh M, Golestani A, Ghazi-Khansari M, Baeeri M, Abdollahi M. Protection by pentoxifylline of malathion-induced toxic stress and mitochondrial damage in rat brain. Hum Exp Toxicol 2010;29:851-64. doi: 10.1177/0960327110363836

170. Shadnia S, Dasgar M, Taghikhani S, Mohammadirad A, Khorasani R, Abdollahi M. Protective effects of alphatocopherol and $\mathrm{N}$-acetyl-cysteine on diazinon-induced oxidative stress and acetylcholinesterase inhibition in rats. Toxicol Mech Methods 2007;17:109-15. doi: 10.1080/15376510600860318

171. Bird SB, Dawson A, Ollis D. Enzymes and bioscavengers for prophylaxis and treatment of organophosphate poisoning. Front Biosci (Scholar Ed). 2010;2:209-20. PMID: 20036941

172. Pourtaji A, Robati RY, Lari P, Hosseinzadeh H, Ramezani $\mathrm{M}$, Abnous K. Proteomics screening of adenosine triphosphate-interacting proteins in the liver of diazinontreated rats. Hum Exp Toxicol 2016;35(10):1084-92. doi: $10.1177 / 0960327115619771$

\section{Štetno djelovanje organofosfornih pesticida na jetra: kratki pregled četrdesetogodišnjeg istraživanja}

Organofosforni pesticidi (OP) imaju veoma široku primjenu, ali i štetno djeluju na jetru pri akutnoj i kroničnoj izloženosti. Ovaj članak daje pregled 40 godina istraživanja djelovanja OP-ova na jetru s namjerom da predloži neke zajedničke mehanizme njihova toksičnog djelovanja na jetru i liječenje. U istraživanjima se izdvaja nekoliko ključnih bioloških procesa koji sudjeluju u hepatotoksičnosti OP-ova, poput narušavanja antioksidacijskoga obrambenog sustava, oksidacijskoga stresa, apoptoze te mitohondrijskoga i mikrosomalnoga metabolizma. Rezultati većine istraživanja potvrdili su da antioksidansi uspješno ublažavaju posljedice oksidacijskoga stresa u jetri. Međutim, gotovo da i nije istražena povezanost između strukture OP-ova i njihove štetnosti odnosno mehanizama djelovanja. Nadamo se da će buduća in vitro i in vivo istraživanja te klinička ispitivanja odgovoriti na preostala pitanja vezana uz mehanizme hepatotoksičnoga djelovanja OP-ova i njegova uspješnoga liječenja. 